Article

\title{
Gauge Theories: From Kaluza-Klein to noncommutative gravity theories
}

\author{
George Manolakos ${ }^{1}\left(\mathbb{D}\right.$, Pantelis Manousselis ${ }^{1}$ and George Zoupanos ${ }^{1,2,3,4, *}$ \\ 1 Physics Department, National Technical University, GR-15780 Athens, Greece \\ 2 Institute of Theoretical Physics, D-69120 Heidelberg, Germany \\ 3 Max-Planck Institut für Physik, Fohringer Ring 6, D-80805 Munchen, Germany \\ 4 Laboratoire d' Annecy de Physique Theorique, 74940 Annecy, France \\ * Correspondence: George.Zoupanos@cern.ch; Tel.: +30-2107723010
}

Received: 28 May 2019; Accepted: 20 June 2019; Published: 2 July 2019

\begin{abstract}
First, the Coset Space Dimensional Reduction scheme and the best particle physics model so far resulting from it are reviewed. Then, a higher-dimensional theory in which the extra dimensions are fuzzy coset spaces is described and a dimensional reduction to four-dimensional theory is performed. Afterwards, another scheme including fuzzy extra dimensions is presented, but this time the starting theory is four-dimensional while the fuzzy extra dimensions are generated dynamically. The resulting theory and its particle content is discussed. Besides the particle physics models discussed above, gravity theories as gauge theories are reviewed and then, the whole methodology is modified in the case that the background spacetimes are noncommutative. For this reason, specific covariant fuzzy spaces are introduced and, eventually, the program is written for both the 3-d and 4-d cases.
\end{abstract}

Keywords: gauge theories; coset space dimensional reduction; fuzzy extra dimensions; higher-dimensional particle physics models; three-dimensional gravity; four-dimensional gravity; noncommutative spaces; fuzzy de Sitter

\section{Introduction}

Throughout the present review, it is evident that, in principle, our ultimate hope is that, as with many theoretical physicists, there is a unification scenario in which all fundamental interactions are included. In this direction, a respectable amount of serious approaches have been considered, with those that elaborate the notion of extra dimensions to be particularly interesting. A consistent framework elaborating the concept of extra dimensions is that of superstring theories [1], with the most promising being the heterotic string [2] (defined in ten dimensions), because of its potential experimental testability. Specifically, the phenomenological aspect of the heterotic string is designated in the resulting Grand Unified Theories (GUTs), containing the gauge group of the Standard Model, which are obtained after compactifying the 10-d spacetime and performing a dimensional reduction of the $E_{8} \times E_{8}$ initial gauge theory. In addition, a few years before the formulation of the superstring theories, the alternative framework of dimensional reduction of higher-dimensional gauge theories was elaborated. This important undertaking, which shared common aims with the one of superstring theories, was initially explored by Forgacs-Manton and Scherk-Schwartz studying the Coset Space Dimensional Reduction (CSDR) [3-5] and the group manifold reduction [6], respectively.

In the higher-dimensional theory, the gauge fields unify the gauge and scalar sector, while, after the dimensional reduction, the resulting 4-d theory comprises a particle spectrum of the surviving components. The above holds for both approaches. Moreover, in the CSDR programme, inclusion of fermions in the higher-dimensional theory gives rise to Yukawa interactions in the resulting 4-d theory. Furthermore, in certain dimensions, there is a way to unify the fields even more if the initial, 
higher-dimensional gauge theory is considered to be $\mathcal{N}=1$ supersymmetric, meaning that the gauge and fermionic fields of the theory are considered in the same vector supermultiplet. In addition, it is worth noting that the CSDR scheme allows the possibility of obtaining chiral 4-d theories $[7,8]$.

In the CSDR context, a couple of elements coming from the heterotic string [2] were incorporated, specifically the dimensionality of the spacetime and the gauge group of the higher-dimensional theory. Therefore, taking into consideration that superstring theories are consistent in ten dimensions, the extra dimensions have to be distinguished from the four observable ones with a compactification of the metric and then the resulting 4-d theory has to be determined. Furthermore, a suitable choice of the compactification manifold could lead to $4-\mathrm{d} \mathcal{N}=1$ supersymmetric theories, aiming for realistic GUTs.

In the procedure of the dimensional reduction of an $\mathcal{N}=1$ supersymmetric gauge theory, a very important and desired property, is the amount of supersymmetry of the initial theory to be preserved in the 4-d one. Demanding this preservation to hold, very good candidates are the compact internal Calabi-Yau (CY) manifolds [9]. However, the moduli stabilization problem that emerged led to the study of flux compactification, in a wider class of internal spaces, the manifolds with SU(3)-structure. In these manifolds, a background non-vanishing, globally defined, spinor is considered. This spinor is covariantly constant with respect to a connection including torsion, while, in the CY case, this holds for a Levi-Civita connection. Here, the nearly-Kähler manifolds class of the SU(3)-structure manifolds is considered [10-32]. Specifically, the class of 6-d homogeneous nearly-Kähler manifolds comprises of the non-symmetric coset spaces $\mathrm{G}_{2} / \mathrm{SU}(3), \mathrm{Sp}(4) / \mathrm{SU}(2) \times \mathrm{U}(1)_{\text {non-max }}, \mathrm{SU}(3) / \mathrm{U}(1) \times \mathrm{U}(1)$ and the group manifold $\mathrm{SU}(2) \times \mathrm{SU}(2)$ [32] (see also [10-31]). It is worth mentioning that, contrary to the CY case, the dimensional reduction of a $10-\mathrm{d} \mathcal{N}=1$ supersymmetric gauge theory over a non-symmetric coset space, leads to 4-d theories which include supersymmetry breaking terms [33-35].

In addition, there exists another appropriate framework, which seems to admit the construction of theories describing physics at Planck scale, in which the property of commutativity of the coordinates is not in force, namely the noncommutative geometry [34,36-78]. This framework is particularly interesting since it provides the possibility of regularizing quantum field theories and, even better, theories equipped with the feature of finiteness can be constructed. However, the construction of quantum field theories on noncommutative spaces proved to be a rather difficult undertaking and problematic ultraviolet behavior has been noticed, too [40-43] (see also [44-46]). Despite that, the framework of noncommutative geometry is considered as a solid ground for the accommodation of particle physics models, formulated as noncommutative gauge theories [47-49] (see also [50-55]).

It is notable that the two frameworks of superstring theories and noncommutative geometry have made contact, since, in M-theory and open string theory, the effective physics on D-branes can be written as a noncommutative gauge theory, in the presence of a non-vanishing background antisymmetric field [56,57]. In addition, the type IIB superstring theory (and others which are related to the type IIB through dualities), in its non-perturbative formulation as a matrix model [58], is in fact a noncommutative theory. In the framework of noncommutativity, Seiberg and Witten [57] made an important contribution, i.e., the map between commutative and noncommutative gauge theories, relating the noncommutative degrees of freedom of a gauge theory to their commutative counterparts. Based on this mapping, important developments were noticed [59-63], for instance the construction of a noncommutative version of Standard Model [64-66], but, unfortunately, its main problem remained unsolved, specifically the number of the free parameters of the model was not achieved to be reduced by extensions of this type.

In the context of the construction of noncommutative particle physics models, particularly interesting are the ones that are related to higher dimensions, which are considered to be noncommutative (fuzzy) [67-78]. This program has the following advantages: (i) exclusion of the ultraviolet/infrared problematic features; (ii) they are renormalizable, in contrast to all known higher-dimensional theories; and (iii) they give the opportunity to begin with an Abelian gauge group in high dimensions and result with a 4-d theory that is non-Abelian, which means an enhancement in the symmetry of the theory. The aspect of renormalizability of such theories was examined from 
the 4-d point of view too, with the starting theory being a 4-d renormalizable $\mathcal{N}=4$ supersymmetric gauge theory and the induction of a symmetry breaking led to a vacuum state, which resembled the results of a dimensional reduction of a higher-dimensional gauge theory where the extra dimensions are considered to be noncommutative (fuzzy) [74,75]. In addition, in the above context, chirality of the fermions of the theory was successfully obtained after an orbifold projection on the spectrum of the initial theory. After the projection and the symmetry breaking, an $\mathcal{N}=1$ supersymmetric, chiral $\mathrm{SU}(3)^{3}$ unified theory is obtained $[74,75]$.

Besides the accommodation of particle physics models, the framework of noncommutative geometry can be employed for the construction of gravity models formulated as noncommutative gauge theories. To comment on this aspect, it is imperative that we recall the correspondence between ordinary gravitational theories in three and four dimensions and ordinary gauge theories [79-91]. The first attempt of this undertaking was made by Utiyama [79], who worked on the description of $4-d$ gravity of General Relativity as a gauge theory of the Lorentz group, $\mathrm{SO}(1,3)$. Since the vielbein were introduced in a rather ambiguous way, the results were not considered to be successful. It was Kibble [80] who took the previous attempt one step further, considering the Poincare group, $\operatorname{ISO}(1,3)$, as the gauge group and, besides the spin connection, the vierbeins were also considered as gauge fields of the theory. However, it had not been definite yet that 4-d gravity could be considered as a gauge theory, since there was no promising candidate of an action for a gauge theory of the Poincare gauge group resembling the Einstein-Hilbert action, without recovering the dynamical part of General Relativity. However, the problem was eventually addressed with the consideration of an $\mathrm{SO}(1,4)$ gauge invariant Yang-Mills action with the simultaneous introduction of a scalar field in the fundamental representation of the gauge group [81] (see also [82-85]). The gauge fixing of the scalar field is responsible for the induction of a spontaneous symmetry breaking resulting to the Einstein-Hilbert action. Therefore, it was eventually achieved to express the gravitational theory of General Relativity in four dimensions as a gauge theory.

Another project contributing in the gauge-theoretic understanding of gravitational interaction is the description of Weyl gravity (and supergravity) as gauge theories of the 4-d conformal group [86,87] (For a textbook see [88].). More explicitly, some of the the gauge fields are identified as the vierbein and spin connection and the translations are related to the general coordinate transformations. Following the standard procedure, the gauge fields and the expressions of the component curvature tensors are obtained. The action is $\mathrm{SO}(2,4)$ gauge invariant of Yang-Mills type and imposition of certain constraints on the curvature tensors along with specific gauge fixing of the fields leads to an action that is in fact the action of Weyl gravity. Therefore, one concludes that Weyl gravity is described as a gauge theory of the conformal group.

Furthermore, Einstein's 3-d gravity is also described as a gauge theory of the 3-d Poincare group, ISO $(1,2)$ (the 3-d de Sitter and anti-de Sitter groups, $\mathrm{SO}(1,3)$ and $\mathrm{SO}(2,2)$, respectively, when the cosmological constant is non-vanishing) [91]. The construction of the 3-d $\operatorname{ISO}(1,2)$ gauge theory admits a Chern-Simons action functional, which is eventually identified as the 3-d Einstein-Hilbert's action. Summing up, the 3-d Einstein's gravitational theory is equivalent to an $\operatorname{ISO}(1,2)$ Chern-Simons gauge theory.

According to the above approaches of gravity as gauge theories, the existence of noncommutative gauge theories [92] could motivate one to consider the above as guideline aiming at noncommutative gravity. Approaches of this type have been formulated before (see, for example, [93-97] and, specifically, for three dimensions employing the Chern-Simons gauge theory formulation, see [98-101]). All above works share a common feature, that of constant noncommutativity (Moyal-Weyl), but also the whole analysis is made using the corresponding $\star$-product and the Seiberg-Witten map [57].

However, gravitational models in the noncommutative framework can be constructed by making use of another type of realization of noncommutative geometries, i.e., the matrix geometries [58,102]. Approaches of this type, specifically Yang-Mills matrix models, have been suggested in the past few years (see [103-113]). For alternative approaches, see the work [39,114,115], as well as in [67-69]. 
In general, formulation of gravity in the framework of noncommutativity implies that Lorentz invariance is broken by the noncommutative deformations. However, there are certain types of deformations which preserve the Lorentz invariance called covariant noncommutative spaces [116,117]. In this spirit, in [118], a noncommutative deformation of a general conformal field theory defined on 4-d dS or AdS spacetime has been considered. Other 4-d approaches can be found in [119-122].

This article includes our contributions in the above aspect of noncommutative gravity. First, we review our approach for 3-d noncommutative gravity as a matrix model [123] (see also [124]), in which the corresponding space is the $\mathbb{R}_{\lambda}^{3}$, introduced in [125] (for the construction of field theories on this space, see also [126]), which is in fact the foliation of the 3-d Euclidean space by fuzzy spheres of different radii. This space admits a natural action of SO(4) [127] and is the group we consider as the gauge group of the theory. Due to noncommutativity, the $\mathrm{SO}(4)$ symmetry is eventually extended to the $\mathrm{U}(2) \times \mathrm{U}(2)$ in a fixed representation, for reasons that we explain explicitly in the corresponding section of the present review. In addition, we formulate the Lorentz analog of the above construction, in which the corresponding noncommutative space we consider is the $\mathbb{R}_{\lambda}^{1,2}$, i.e., the foliation of the 3-d Minkowski spacetime by fuzzy hyperboloids of ref. [128]. Again, the initial symmetry, SO(1,3), has to be enlarged to $G L(2, \mathbb{C})$ in a fixed representation. In both cases, the action is given in terms of a functional of Chern-Simons type and variation leads to the equations of motion. In addition, the commutative limit is studied and the results of the 3-d Einstein's gravity are recovered successfully.

Our second contribution in the noncommutative gravity field is the formulation of a 4-d gravity model as a noncommutative gauge theory [129]. In the spirit of Yang's pioneering work [117] and inspired by the work of Heckman-Verlinde [118], we define a fuzzy version of the 4-d de Sitter space, which constitutes a covariant fuzzy space. Noncommutative coordinates of this space are identified as part of the generators of the algebra of the symmetry group, $\mathrm{SO}(1,4)$, with proper modifications taking place to preserve Lorentz invariance. Then, for the same reasons as in the 3 -d case, the final gauge group is determined to be the $\mathrm{SO}(1,5) \times \mathrm{U}(1)$. The corresponding gauge fields are identified and, following the standard procedure, the transformations of the fields and their corresponding component curvature tensors are obtained. Then, for the dynamical part, an action of Yang-Mills type is considered and its symmetry gets broken after the imposition of constraints on the curvature tensors and field gauge fixing. The commutative limit of our model produces expressions that are related to the ones of conformal gravity in its gauge-theoretic approach.

The present article is divided into two main parts, the first one dealing with particle physics models using extra dimensions (fuzzy or not), while the second one dealing with gravity models in the noncommutative framework. In both parts, necessary information is provided in order to render this review self-contained.

\section{Coset Space Dimensional Reduction of a Higher-Dimensional Theory}

The first attempt in the concept of the dimensional reduction of a higher-dimensional theory, in which 4-d theories are obtained starting from a unified theory, was that of Kaluza-Klein [130,131]. Specifically, the idea was to consider a pure 5-d gravitational theory and reduce it to four dimensions resulting to a $\mathrm{U}(1)$ gauge theory (electromagnetism) coupled to gravity. Later, attention was drawn again in this scheme after the realization that non-Abelian group structures appear in a natural way by the extension of the spacetime [132-134] (for reviews, see [135-137]). Taking into account that the total, higher-dimensional spacetime can be expressed as a Cartesian product, $M^{D}=M^{4} \times B$, where $B$ is a compact Riemannian manifold with isometries parameterized by the group $S$, then performing a dimensional reduction of the initial theory leads to a 4-d theory in which gravity is coupled to a Yang-Mills theory with a gauge group that includes the $S$ group, along with a set of scalar fields. The main virtues of this scheme are the geometrical unification of gravity and other interactions described by gauge theories and the origin of the gauge-theoretic description of the other interactions. Besides the above encouraging features, there are also others that are not welcome. For instance, there is no classical ground state corresponding to the structure of $M^{D}$ as a direct product. 
The Einstein equations of the gravitational theory in $D$ dimensions lead to vanishing Ricci tensor, which is unacceptable, as the Ricci tensor of a compact space with non-Abelian isometry cannot be identically zero. Therefore, it is concluded that the extra dimensions do not admit a physical interpretation. Besides that, another striking problem is that the inclusion of fermions in the initial theory does not lead to chiral fermions in the low-energy theory, after the dimensional reduction, which means that it is not possible to result with realistic models [138]. However, considering an appropriate particle spectrum, in particular Yang-Mills fields, in the initial theory, it is possible to end up with a stable classical ground state, but also to obtain massless fermions and parity violation in the fermion sector in four dimensions $[8,139]$. Therefore, one is led to introduce Yang-Mills fields in higher dimensions.

Taking into consideration that the gravitational interaction is negligible at the low-energy phenomena, one can start with a Yang-Mills theory defined on the higher-dimensional spacetime, $M^{D}=M^{4} \times B$ and result with a Yang-Mills-Higgs 4-d theory, achieving a potential unification of the interactions, as well as a unification of gauge and Higgs sectors. A straightforward and rather crude way to perform a dimensional reduction of a higher-dimensional gauge theory is to consider that the fields consisting the spectrum of the initial theory do not depend on the extra coordinates. Under this consideration, the Lagrangian would be independent of the extra coordinates, too. Nevertheless, one could consider that the extra dimensions form a coset space, $B=S / R$, allowing the fields to depend on the extra coordinates in a non-trivial way, such that an S-transformation changes the Lagrangian in such a way that is compensated by a gauge transformation. Therefore, independence of the Lagrangian of the extra coordinates is achieved because of its gauge invariance. In fact, the above consideration is the core and starting point of the Coset Space Dimensional Reduction (CSDR) scheme [3-5]. In the following section, we review its basic concepts and highlight the most crucial technical points.

\subsection{CSDR Constraints}

Let us consider a higher-dimensional Yang-Mills theory of gauge group $G$, coupled to fermions, defined on $M^{D}$, where $\mathrm{D}$ is the dimension of the spacetime, with $g_{M N}$ being the metric tensor. Considering that fermions are included in the theory, the corresponding action would be:

$$
A=\int d^{4} x d^{d} y \sqrt{-g}\left[-\frac{1}{4} \operatorname{Tr}\left(F_{M N} F_{K \Lambda}\right) g^{M K}{ }_{g} N \Lambda+\frac{i}{2} \bar{\psi} \Gamma^{M} D_{M} \psi\right],
$$

where $D_{M}=\partial_{M}-\theta_{M}-A_{M}$ is the covariant derivative, with $\theta_{M}=\frac{1}{2} \theta_{M N \Lambda} \Sigma^{N \Lambda}$ the spin connection of $M^{D}, F_{M N}=\partial_{M} A_{N}-\partial_{N} A_{M}-\left[A_{M}, A_{N}\right]$ the field strength tensor of $G$ and $M, N, K, \Lambda=1 \ldots D$. In addition, $A_{M}$ and $\psi$ are D-dimensional symmetric gauge and spinor fields, respectively. In general, the fermions of the theory could be in arbitrary representation $F$ of $G$. However, in the case the theory is considered to be supersymmetric, then fermions should be accommodated into the same representation of the gauge fields, composing a vector supermultiplet.

Let us consider the Killing vectors, $\xi_{A}^{\alpha}, A=1, \ldots, \operatorname{dim} S$ and $\alpha=\operatorname{dim} R+1, \ldots, \operatorname{dim} S$, of $S / R$ and the gauge transformation, $W_{A}$, corresponding to $\xi_{A}$. Considering the various fields of the theory to be symmetric, then, for scalar $\phi$, vector $A_{\alpha}$ and spinor $\psi$ fields on $S / R$, the following constraint equations are produced:

$$
\begin{gathered}
\delta_{A} \phi=\xi_{A}^{\alpha} \partial_{\alpha} \phi=D\left(W_{A}\right) \phi, \\
\delta_{A} A_{\alpha}=\xi_{A}^{\beta} \partial_{\beta} A_{\alpha}+\partial_{\alpha} \xi_{A}^{\beta} A_{\beta}=\partial_{\alpha} W_{A}-\left[W_{A}, A_{\alpha}\right], \\
\delta_{A} \psi=\xi_{A}^{\alpha} \partial_{\alpha} \psi-\frac{1}{2} G_{A b c} \Sigma^{b c} \psi=D\left(W_{A}\right) \psi,
\end{gathered}
$$

where the gauge transformation, $W_{A}$, depends exclusively on the coordinates $y$ of the extra dimensions and, in addition, $D\left(W_{A}\right)$ denotes the specific representation of the gauge transformation for each one of the fields of the theory. Solving the above constraints and taking into consideration that the fields are considered to be symmetric, Equations (2)-(4), one results with the corresponding unconstrained fields 
in four dimensions, as well as with the gauge group parameterizing the gauge symmetry of the 4-d theory $[3,4]$. Now, further procession of the above constraints gives the following information about the 4-d theory: From Equation (2), the splitting of the gauge fields $A_{M}$ (defined on $M_{D}$ ) into its $M^{4}$ and $S / R$ components, as $\left(A_{\mu}, A_{\alpha}\right)$, is obtained. The solution of the second constraint, Equation (3), renders the 4 -d vector field, $A_{\mu}$, to be independent of the coordinates of the coset space. In addition, an additional understanding from this constraint is that the 4-d gauge fields commute with the generators of the subgroup $R$ of $G$. This commutation relation leads to the fact that the remaining gauge symmetry, $H$, should be the subgroup of $G$, which also commutes with the subgroup $R$. Therefore, the remaining gauge symmetry is the centralizer of $R$ in $G$, namely $H=C_{G}\left(R_{G}\right)$. The $A_{\alpha}(x, y)$ component of the gauge field is transforming as a set of scalars in the 4-d theory denoted as $A_{\alpha}(x, y) \equiv \phi_{\alpha}(x, y)$ and are understood as intertwining operators, connecting the induced representations of $R$ acting on $G$ and the coset space, $S / R$. The representation of the $4-d$ scalar fields is obtained by the following decomposition of $G$ according to the following embedding:

$$
G \supset R_{G} \times H, \quad \operatorname{adj} G=(\operatorname{adjR}, 1)+(1, \operatorname{adj} H)+\sum\left(r_{i}, h_{i}\right),
$$

and decomposition of $S$ under $R$ :

$$
S \supset R, \quad \text { adj } S=\operatorname{adj} R+\sum s_{i} .
$$

The above scheme manifests the representation of the scalars because, for every $r_{i}, s_{i}$ identical representations, there is a remaining scalar multiplet, which transforms under the $h_{i}$ representation of the subgroup $H$. Every other scalar field not included in the $h_{i}$ vanishes.

Now, the solution of the third constraint, Equation (4), gives us insight on the spinorial part of the the 4-d theory, with the analysis on this constraint being similar to the previous one $[4,7,8,140-143]$. As expected, the 4-d spinors do not depend on the coordinates of the coset space and act as operators which intertwine between the induced representations of $R$ in $S O(d)$ and $G$. The representation $f_{i}$ of the subgroup $H$, i.e., the remnant gauge symmetry in four dimensions into which the fermions eventually fall, is obtained after the decomposition of the initial representation $F$ of $G$ of the fermions under the $R_{G} \times H$ as follows:

$$
G \supset R_{G} \times H, \quad F=\sum\left(r_{i}, f_{i}\right),
$$

and the spinor of $S O(d)$ under $R$ :

$$
S O(d) \supset R, \quad \sigma_{d}=\sum \sigma_{j} .
$$

In the 4-d theory, for the identical representations $r_{i}$ and $\sigma_{i}$, there exists a multiplet, $f_{i}$, of spinor fields. Had the fermions been considered as Dirac fermions in the initial, higher-dimensional theory, then there would be no chance for chiral fermions in the resulting, 4-d theory. However, imposition of the Weyl condition on the (necessarily even) higher-dimensional theory would lead to chiral fermions in the 4-d theory. Moreover, specifically in the case in which the dimension of the initial theory is $D=2 n+2$ and fermions are accommodated into the adjoint representation, imposition of the Weyl condition would produce two sets of chiral fermions with the same quantum numbers under the 4-d gauge group, $H$. Furthermore, additional imposition of the Majorana condition cancels the above duplicate of the fermionic spectrum. In the case of interest, i.e., of $D=4 n+2$ dimensions, the Weyl and Majorana conditions are compatible, which means that they can be imposed at the same time. 


\subsection{The 4-d Effective Action}

The next step is the determination of the effective action of the resulting 4-d theory. First, the higher-dimensional space, $M^{D}$, is compactified to $M^{4} \times S / R$, with $S / R$ being a compact coset space. Due to the above compactification, the metric of the space $M^{D}$ takes the following form:

$$
g^{M N}=\left(\begin{array}{cc}
\eta^{\mu \nu} & 0 \\
0 & -g^{a b}
\end{array}\right)
$$

where the blocks of the above matrix are the (mostly negative) metric of the 4-d Minkowski spacetime and $g^{a b}$, which is the metric of the corresponding coset space. Replacing the above expression of the metric, Equation (9), into the initial Yang-Mills action, Equation (1), and taking into consideration the information from the solutions of the constraints of the fields, Equations (2)-(4), the effective action of the 4-d theory would be:

$$
A=C \int d^{4} x\left[-\frac{1}{4} F_{\mu v}^{t} F^{t \mu v}+\frac{1}{2}\left(D_{\mu} \phi_{\alpha}\right)^{t}\left(D^{\mu} \phi^{\alpha}\right)^{t}+V(\phi)+\frac{i}{2} \bar{\psi} \Gamma^{\mu} D_{\mu} \psi-\frac{i}{2} \bar{\psi} \Gamma^{a} D_{a} \psi\right]
$$

where $D_{\mu}=\partial_{\mu}-A_{\mu}$ and $D_{a}=\partial_{a}-\theta_{a}-\phi_{a}$, with $\theta_{a}=\frac{1}{2} \theta_{a b c} \Sigma^{b c}$ the connection of the space and $C$ the volume of the coset space. The scalar potential, $V(\phi)$, is found to be:

$$
V(\phi)=-\frac{1}{4} g^{a c} g^{b d} \operatorname{Tr}\left(f_{a b}{ }^{C} \phi_{C}-\left[\phi_{a}, \phi_{b}\right]\right)\left(f_{c d}{ }^{D} \phi_{D}-\left[\phi_{c}, \phi_{d}\right]\right),
$$

where $A=1, \ldots, \operatorname{dim} S$ and $f_{a b}{ }^{C}, f_{c d}^{D}$ are the structure constants of the Lie algebra of the isometry group $S$ of the coset space. From the expressions of the constraints of the fields, Equations (2) and (3), it is implied that the scalar fields, $\phi_{a}$, satisfy the following relation:

$$
f_{a i}^{D} \phi_{D}-\left[\phi_{a}, \phi_{i}\right]=0,
$$

where $\phi_{i}$ are the generators of $R_{G}$. Therefore, some of the scalars will be filtered out, while others will survive the dimensional reduction and will be identified as the Higgs fields of the 4-d theory. The scalar potential, $V(\phi)$, is a gauge invariant quartic polynomial. Then, minimization of the potential leads to the vacuum of the theory and the final gauge symmetry is determined [144-151]. Although generally it is a rather difficult task to obtain the vacuum and the remnant gauge symmetry, in the specific case in which $S$ has an isomorphic image $S_{G}$ in $G$, the 4-d gauge group $H$ is spontaneously broken to a subgroup, $K$, which is the centralizer of $S_{G}$ in the initial higher-dimensional gauge group, G [4,144-151]:

$$
\begin{array}{ll}
G \supset & S_{G} \times K \\
& \cup \cap \\
G \supset & R_{G} \times H .
\end{array}
$$

If the coset space is symmetric $\left(f_{a b}^{c}=0\right)$, the potential of the 4-d gauge theory always takes a form in which it breaks spontaneously [4]. In addition, application of the above scheme produces supermassive fermions [152], as it happens in the Kaluza-Klein theory, which generally is not a desired outcome. However, an interesting exception is the example that follows.

Let us now apply the above program on a higher-dimensional $\mathcal{N}=1, E_{8}$ supersymmetric Yang-Mills theory. The initial 10-d spacetime is compactified to $M^{4} \times S U(3) / U(1) \times U(1)$, where $S U(3) / U(1) \times U(1)$ is a nearly-Kähler manifold. The 4-d gauge group, $H$, is obtained by the decomposition of the $E_{8}$ under $R=U(1) \times U(1)$, since, as found from the solution of the constraint in Equation (3), the subgroup, $H$, is the centralizer of $R=U(1) \times U(1)$ in $G=E_{8}$ :

$$
H=C_{E_{8}}\left(U(1)_{A} \times U(1)_{B}\right)=E_{6} \times U(1)_{A} \times U(1)_{B}
$$


since $E_{8} \supset E_{6} \times S U(3) \supset E_{6} \times U(1)_{A} \times U(1)_{B}$. The resulting 4-d theory is an $\mathcal{N}=1, E_{6}$ GUT, with additional $U(1)_{A}, U(1)_{B}$ global symmetries. The determination of the potential is obtained after tedious calculations. For the procedure and the explicit expression, see [35]. In the expression of the potential, there are contributions from the $F-$ and $D$ - terms, but also scalar masses and trilinear scalar terms are also included. In addition, gaugino gains mass in which the torsion contributes. It is remarkable that in the CSDR scheme the supersymmetry breaking sector is automatically retrieved, without additional considerations. The 4-d unified gauge theory, $E_{6}$, which is obtained after the CSDR, can be broken employing the Wilson flux mechanism (for details, see [30,31]), to an $\mathcal{N}=1$, chiral $\operatorname{SU}(3)^{3}$ theory. Further breaking of this gauge theory leads to an extended version of the MSSM.

\section{Fuzzy Spaces}

For reasons explained in the Introduction, noncommutative geometry is a particularly interesting framework in which particle physics models can be built. A specific class of noncommutative spaces is that of fuzzy spaces, which are noncommutative analogs of ordinary spaces preserving the isometries. In the matrix algebraic formulation, fuzzy spaces are in fact matrix approximations of continuous manifolds. For the time being, we write the description of the most typical case of fuzzy spaces, i.e., the fuzzy sphere [38], and also give some information about the construction of gauge theories on it. This is sufficient for our current purpose, i.e., the construction of particle physics models with fuzzy extra dimensions. In a later section, we give details of more such spaces, generalizing the case of the fuzzy sphere, used in the construction of noncommutative gravity models.

\subsection{The Fuzzy Sphere}

To define the fuzzy sphere, first, let us recall a few details about the definition of the ordinary sphere, $S^{2}$. The ordinary sphere can be defined as an embedding into the 3-d Euclidean space, $\mathbb{R}^{3}$, with points on it being labeled by a Cartesian coordinate system, $x_{a}, a=1,2,3$ obeying the following constraint equation:

$$
\sum_{a=1}^{3} x_{a}^{2}=r^{2},
$$

where $r$ is the radius of the sphere. The isometry group of the sphere is the $\mathrm{SO}(3)$, which is the parameterization of rotational symmetry in three-dimensions and is generated by the three operators of angular momentum, $L_{a}=-i \epsilon_{a b c} x_{b} \partial_{c}$. Alternatively, these generators can be written with respect to the spherical coordinates, $\theta, \phi$. The corresponding Laplace operator is defined on the sphere by the relation $L^{2}=-R^{2} \Delta_{S^{2}}$ and its eigenfunctions are the spherical harmonics, $Y_{l m}(\theta, \phi)$, defined as:

$$
Y_{l}^{m}(\theta, \phi)=N e^{i m \phi} P_{l}^{m}(\cos \theta)
$$

where $P_{l}^{m}$ denote the Legendre polynomials. Spherical harmonics satisfy the following orthogonality and normalization condition:

$$
\int d \Omega Y_{l m}^{+} Y_{l^{\prime} m^{\prime}}=\delta_{l l^{\prime}} \delta_{m m^{\prime}}
$$

Moreover, spherical harmonics consist a complete and orthogonal set of functions, therefore, any function defined on the sphere can be written as an expansion:

$$
f(\theta, \phi)=\sum_{l=0}^{\infty} \sum_{m=-l}^{l} a_{l m} Y_{l m}(\theta, \phi),
$$

where the coefficients $a_{l m}$ are complex numbers.

Let us now consider the case in which the algebra of the spherical harmonics is truncated, which means that $l$ has a maximum, finite value. If we consider two functions on the sphere, expanded on this finite set of spherical harmonics, then their product would give terms that exceed the maximum 
value of $l$, therefore, the algebra is not closed under the multiplication operation. An effective way to recover closure under multiplication is to consider a different kind of product, more specifically a noncommutative one. Therefore, it is understood that noncommutativity under multiplication is the cost of the truncation of the algebra of functions. The most obvious choice is the matrix product, which means that functions are considered to be matrices. The above space is defined as the fuzzy sphere.

A straightforward formulation of the fuzzy sphere is achieved by adopting the finite-dimensional noncommutative matrix algebra. Let us consider the three $(N+1)$-dimensional matrices $J_{a}, a=1,2,3$ that are an irreducible representation of $(N+1)$ dimension of the $\mathrm{SU}(2)$ generators, which satisfies the following commutation relation:

$$
\left[J_{a}, J_{b}\right]=i \epsilon_{a b c} J_{c} .
$$

The Casimir operator in this $(N+1)$-dimensional irreducible representation is given by:

$$
J^{2}=J_{1}^{2}+J_{2}^{2}+J_{3}^{2}=\frac{N}{2}\left(\frac{N}{2}+1\right) \mathbf{1}_{N+1} .
$$

Thus, the fuzzy sphere (at $N$ level of fuzziness) is a fuzzy space with coordinates, $\hat{X}_{a}, a=1,2,3$ represented by $(N+1) \times(N+1)$ Hermitian matrices, which are related through a proportionality constant to the generators of $\mathrm{SU}(2)$ in the $(N+1)$-dimensional irreducible representation, namely:

$$
\hat{X}_{a}=\kappa J_{a} .
$$

The coordinates of the fuzzy sphere that parameterizes its points, obey the following radius constraint:

$$
\sum_{a=1}^{3} \hat{X}_{a} \hat{X}_{a}=\hat{X}_{1}^{2}+\hat{X}_{2}^{2}+\hat{X}_{3}^{2}=r^{2}
$$

where $r$ is the fuzzy sphere radius. Employing the definition of the Casimir operator, Equation (20), and the expression of the coordinates being proportional to the generators, Equation (21), the proportionality constant, $\kappa$, is obtained:

$$
\kappa=\frac{r}{\sqrt{\frac{N}{2}\left(\frac{N}{2}+1\right)}}=\lambda_{N} r
$$

where $\lambda_{N}=\frac{1}{\sqrt{\frac{N}{2}\left(\frac{N}{2}+1\right)}}$. Therefore, the coordinate matrices can now be written as:

$$
\hat{X}_{a}=\kappa J_{a}=\frac{r}{\sqrt{\frac{N}{2}\left(\frac{N}{2}+1\right)}} J_{a}=\lambda_{N} r J_{a} .
$$

In addition, the commutation relation of the $J_{a}$ generators of $S U(2)$, Equation (19), leads to the commutation relation of the coordinates:

$$
\left[\hat{X}_{a}, \hat{X}_{b}\right]=i \kappa \epsilon_{a b c} \hat{X}_{c}=i \lambda_{N} C_{a b c} \hat{X}_{c}
$$

where Equation (23) has been considered and $C_{a b c}$ is the rescaled Levi-Civita symbol, explicitly, $C_{a b c}=r \epsilon_{a b c}$. The coordinate matrices, $\hat{X}_{a}$, admit an alternative description in terms of the anti-Hermitian matrices, $X_{a}$, which are defined by the following relation:

$$
X_{a}=\frac{1}{i \kappa r} \hat{X}_{a}=\frac{1}{i r} J_{a}
$$


Accordingly, their commutation relation, Equation (25), and the corresponding constraint of the radius, Equation (22), are now expressed as:

$$
\left[X_{a}, X_{b}\right]=C_{a b c} X_{c} \quad \text { and } \quad \sum_{a=1}^{3} X_{a} X_{a}=-\frac{\lambda_{N}^{-2}}{r^{2}}
$$

where $C_{a b c}$ is now identified as $C_{a b c}=\frac{\epsilon_{a b c}}{r}$. Obviously, the algebra of the fuzzy sphere is described by both definitions on equal footing.

It is worth noting that, besides functions, spinors can also be defined on the fuzzy sphere [67-69]. Furthermore, studies on the differential geometry of more higher-dimensional fuzzy spaces, e.g., the fuzzy $C P^{M}$ [67-69], have been carried out.

Concluding, integration on the fuzzy sphere is performed by the following mapping:

$$
\frac{1}{4 \pi} \int d \Omega \rightarrow \frac{1}{N} \operatorname{Tr}_{N}
$$

Differential calculus on a fuzzy sphere is 3-d and SU(2) covariant [67-69]. Derivation is performed by the commutator of an element of the algebra on the fuzzy sphere acting on a function $\hat{f}$. Therefore, derivation along the coordinate $X_{a}$ is given by:

$$
e_{a}(\hat{f})=\left[X_{a}, \hat{f}\right] .
$$

The above definitions are particularly essential for the construction of gauge theories on the fuzzy sphere, which is discussed in the next section.

\subsection{Gauge Theory on a Fuzzy Sphere}

Let us now consider a field $\phi\left(X_{a}\right)$ on the fuzzy sphere, expressed in terms of powers of $X_{a}$ [92]. An infinitesimal gauge transformation of $\phi\left(X_{a}\right)$ under a gauge group, $G$, is:

$$
\delta \phi(X)=\lambda(X) \phi(X),
$$

where $\lambda(X)$ is the gauge transformation parameter. If $\lambda(X)$ is a function of $X_{a}$, then it is an infinitesimal Abelian transformation and $G=U(1)$, while, if $\lambda(X)$ is a $P \times P$ Hermitian matrix, then the transformation is non-Abelian and the gauge group is $G=U(P)$. In addition, it holds that the coordinates are invariant under a gauge transformation of the internal symmetry, namely $\delta X_{a}=0$. Now, the gauge transformation of the field multiplied by the coordinate is not covariant, namely:

$$
\delta\left(X_{a} \phi\right)=X_{a} \lambda(X) \phi,
$$

since, in general:

$$
X_{a} \lambda(X) \phi \neq \lambda(X) X_{a} \phi
$$

Motivated by the construction of ordinary gauge theories, in which covariant derivative is introduced for similar reasons, in the fuzzy case, the covariant coordinate, $\phi_{a}$, is introduced as:

$$
\delta\left(\phi_{a} \phi\right)=\lambda \phi_{a} \phi
$$

which is satisfied when:

$$
\delta\left(\phi_{a}\right)=\left[\lambda, \phi_{a}\right] .
$$

In addition, again motivated by ordinary gauge theories, the following definition of the covariant coordinate is introduced:

$$
\phi_{a} \equiv X_{a}+A_{a}
$$


in which $A_{a}$ is interpreted as the gauge connection of the noncommutative gauge theory. Starting from Equations (34) and (35), the gauge transformation of $A_{a}$ is obtained:

$$
\delta A_{a}=-\left[X_{a}, \lambda\right]+\left[\lambda, A_{a}\right] .
$$

The above expression designates the role of $A_{a}$ as a gauge field. The next step is to define the field strength tensor, $F_{a b}$ :

$$
F_{a b} \equiv\left[X_{a}, A_{b}\right]-\left[X_{b}, A_{a}\right]+\left[A_{a}, A_{b}\right]-C_{a b}^{c} A_{c}=\left[\phi_{a}, \phi_{b}\right]-C_{a b}^{c} \phi_{c}
$$

which transforms in a covariant way:

$$
\delta F_{a b}=\left[\lambda, F_{a b}\right] .
$$

In the next section, the above toolkit is employed for the construction of particle physics gauge theories, in which fuzzy extra dimensions are included.

\section{Dimensional Reduction of a Higher-Dimensional Theory with Fuzzy Extra Dimensions}

In this section, a dimensional reduction of a higher-dimensional theory with fuzzy extra dimensions is presented, realized in the most straightforward way. Then, a fuzzy extension of the Coset Space Dimensional Reduction is briefly discussed.

\subsection{Ordinary Fuzzy Dimensional Reduction}

Let us consider a higher-dimensional gauge theory, $G=U(P)$, defined on the spacetime $M^{4} \times(S / R)_{F}$, with $(S / R)_{F}$ a fuzzy coset space. The corresponding Y-M action is the following:

$$
\mathcal{S}_{Y M}=\frac{1}{4 g^{2}} \int d^{4} x k \operatorname{Trtr}_{G} F_{M N} F^{M N}
$$

where $\operatorname{tr}_{G}$ is the trace associated with the algebra of $G$ and $k \operatorname{Tr}$ stands for the integration operation over the fuzzy coset (Parameter $k$ is related to the size of the fuzzy coset space, generalizing the $k$ parameter encountered in the case of the fuzzy sphere.) and $F_{M N}$ the field strength tensor of the higher-dimensional gauge theory, containing terms related to the 4-d Minkowski spacetime as well as to the fuzzy coset, namely $\left(F_{\mu v}, F_{\mu a}, F_{a b}\right)$. The mixed and fuzzy part of $F_{M N}$ are given as follows:

$$
\begin{aligned}
F_{\mu a} & =\partial_{\mu} \phi_{a}+\left[A_{\mu}, \phi_{a}\right]=D_{\mu} \phi_{a} \\
F_{a b} & =\left[X_{a}, A_{b}\right]-\left[X_{b}, A_{a}\right]+\left[A_{a}, A_{b}\right]-C_{b a}^{c} A_{a c}
\end{aligned}
$$

where $\phi_{a}$ is the corresponding covariant coordinate. Replacing the above expressions of the field strength components into the initial action, Equation (39), it takes the following form:

$$
\mathcal{S}_{Y M}=\int d^{4} x \operatorname{Trtr}_{G}\left(\frac{k}{4 g^{2}} F_{\mu \nu}^{2}+\frac{k}{2 g^{2}}\left(D_{\mu} \phi_{a}\right)^{2}-V(\phi)\right),
$$

where $V(\phi)$ is identified as the potential, originating from the $F_{a b}^{2}$ term:

$$
\begin{aligned}
V(\phi) & =-\frac{k}{4 g^{2}} \operatorname{Trtr}_{G} \sum_{a b} F_{a b} F_{a b} \\
& =-\frac{k}{4 g^{2}} \operatorname{Trtr}_{G}\left(\left[\phi_{a}, \phi_{b}\right]\left[\phi^{a}, \phi^{b}\right]-4 C_{a b c} \phi^{a} \phi^{b} \phi^{c}+2 R^{-2} \phi^{2}\right) .
\end{aligned}
$$


The expression of the action in Equation (40) is naturally interpreted as a 4-d action. In that sense, let $\lambda\left(x^{\mu}, X^{a}\right)$ be an infinitesimal gauge parameter of $G=U(P)$. However, this transformation parameter can be regarded as a gauge transformation of a gauge theory constructed on $M^{4}$ :

$$
\lambda\left(x^{\mu}, X^{a}\right)=\lambda^{I}\left(x^{\mu}, X^{a}\right) \mathcal{T}^{I}=\lambda^{h, I}\left(x^{\mu}\right) \mathcal{T}^{h} \mathcal{T}^{I},
$$

where $\mathcal{T}^{I}$ are the Hermitian generators of the initial gauge group $\mathrm{U}(\mathrm{P})$ of the higher-dimensional theory and $\lambda^{I}\left(x^{\mu}, X^{a}\right)$ are $\mathrm{N} \times \mathrm{N}$ anti-Hermitian matrices. Therefore, they can be written as $\lambda^{I, h}\left(x^{\mu}\right) \mathcal{T}^{h}$, where $\mathcal{T}^{h}$ are the anti-Hermitian generators of a $\mathrm{U}(\mathrm{N})$ group and $\lambda^{I, h}\left(x^{\mu}\right), h=1, \ldots, N^{2}$, are identified as the Kaluza-Klein modes of $\lambda^{I}\left(x^{\mu}, X^{a}\right)$. The fields on the right hand side of Equation (42) can be naturally viewed as a field valued in the Lie algebra of the tensor product of the two unitary groups, $\mathrm{U}(\mathrm{N}) \times \mathrm{U}(\mathrm{P})$, which is in fact the algebra of the group $\mathrm{U}(\mathrm{NP})$. The same consideration also applies for the gauge field $A_{v}$ as follows:

$$
A_{v}\left(x^{\mu}, X^{a}\right)=A_{v}^{I}\left(x^{\mu}, X^{a}\right) \mathcal{T}^{I}=A_{v}^{h, I}\left(x^{\mu}\right) \mathcal{T}^{h} \mathcal{T}^{I},
$$

which can be seen as a gauge field on 4-d Minkowski spacetime, valued in the algebra of U(NP). A similar consideration can be applied for the scalars, too.

Despite the simplicity of the above dimensional reduction, a very important feature becomes manifest, i.e., the enlargement of the initial gauge symmetry of the higher-dimensional theory to the 4-d one. More specifically, this means that the starting gauge group can be considered to be Abelian and result with a non-Abelian gauge group in four dimensions, due to the enlargement of the symmetry. However, the scalars of the 4-d theory are in the adjoint representation, which means that they are incapable of triggering the electroweak symmetry breaking. To avoid this undesirable feature, a more elaborate scheme of dimensional reduction must be employed.

\subsection{Fuzzy CSDR}

In this section, we briefly present a fuzzy extension of the Coset Space Dimensional Reduction program presented above. The main concept remains the same, i.e., the non-trivial dependence of the fields on the extra-dimensional coordinates, but since the coset space over which the reduction is performed is now fuzzy, modifications on the constraints are expected to be applied [67-69] (see also [153]).

The common ground on both scenarios is that, since fuzzy spaces preserve the isometries of the corresponding manifold, the fuzzy coset considered has the same gauge group of symmetries as its non-fuzzy original space. In addition, gauge couplings defined on both kinds of spaces share the same dimensionality. Now, in the CSDR procedure, we explain that compactification of the space induces a symmetry breaking, resulting with gauge symmetry, $H$, in the 4-d theory, where $H$ is the centralizer of $R$ into $G$. Then, spontaneous symmetry breaking due to the presence of the Higgs scalars led to further reduction of the symmetry to the $K$ gauge group, which was obtained as the centralizer of $S$ into $G$. In the fuzzy CSDR case, the intermediate stage of the 4- $d$ theory being governed by the $H$ symmetry is skipped. In other words, the vacuum and its symmetry in four dimensions is obtained directly with the solutions of the corresponding constraints. Therefore, in the 4-d theory, the scalars that are present are identified as the physical Higgs scalars. Accordingly, the symmetry breaking taking place leads to massive fermions and Yukawa interactions (Yukawa sector). Another difference between the two programs is related to the feature encountered in the previous subsection, in which the straightforward dimensional reduction over fuzzy coset spaces is described, i.e., the choice of an Abelian gauge theory in high dimensions leads to non-Abelian gauge symmetry in four dimensions. Another very welcome feature accompanying the fuzzy CSDR is that the higher-dimensional theory considered as well as the resulting 4-d one are actually renormalizable (see [67-69]). 


\section{Orbifolds and Fuzzy Extra Dimensions}

In this section, a particle physics model using fuzzy extra dimensions leading to low-energy theories that include chiral fermions $[74,75]$ is reviewed. The renormalizability of such higher-dimensional theories is emphasized, following a reverse procedure, i.e., starting with a 4-d renormalizable theory (The whole idea shares some similarities with the idea of dimensional deconstruction, introduced earlier [154,155] (see also [156]).) [70-73]. More explicitly, a renormalizable 4-d $\mathcal{N}=4$ supersymmetric Yang-Mills $\mathrm{SU}(3 \mathrm{~N})$ gauge theory is considered, along with an appropriate particle spectrum of scalars and fermions. Application of an orbifold projection (similar to the one in [157]) leads to reduction of the supersymmetry and the gauge symmetry and filters out some of the fields of the initial theory. Then, symmetry breaking leads to certain vacuum, which can be interpreted as a reduced higher-dimensional theory with fuzzy extra dimensions. The resulting theory is a Grand Unified Theory, which can be further broken to an extension of MSSM (This reverse procedure aims also at proving that it is not necessary to consider an Abelian gauge theory that is higher-dimensional, with the non-Abelian gauge theory structure emerging from fluctuations of the coordinates [158-160].).

Earlier, attempts to include fermions were made with the best model achieved (for some time) involving mirror fermions in bifundamental representations of the gauge group in the low-energy regime [72,73] (Although mirror fermions are not incompatible with phenomenology [161], exactly chiral fermions are preferred.).

\section{1. $\mathcal{N}=4$ SYM Field Theory and $\mathbb{Z}_{3}$ Orbifolds}

Let us first consider an $\mathcal{N}=4$ supersymmetric Yang-Mills $\mathrm{SU}(3 \mathrm{~N})$ gauge theory defined on the 4-d Minkowski spacetime. The particle content of the theory is given in terms of $\mathcal{N}=1$ supersymmetry consisting of an $\mathrm{SU}(3 \mathrm{~N})$ gauge supermultiplet and three adjoint chiral supermultiplets $\Phi^{i}, i=1,2,3$. The fields composing the above supermultiplets are the gauge fields, $A_{\mu}, \mu=1, \ldots, 4$, six adjoint real scalar fields $\phi^{a}, a=1, \ldots, 6$ and four adjoint Weyl fermions $\psi^{p}, p=1, \ldots, 4$. Under the $\mathrm{SU}(4)_{R} R$-symmetry of the theory, the scalars and Weyl fermions belong to the 6 and 4 representations, respectively, while the gauge bosons belong to the trivial representation (singlets). On the above theory, an orbifold projection is performed, parameterized by the $\mathbb{Z}_{3}$ discrete group, which is viewed as a subgroup of $S U(4)_{R}$. The $\mathbb{Z}_{3}$ can be embedded into $S U(4)_{R}$ in various, non-equivalent ways, since the choice of embedding determines the amount of the remnant supersymmetry [157]:

- Maximal embedding of $\mathbb{Z}_{3}$ into $\mathrm{SU}(4)_{R}$ is excluded since it leads to vanishing supersymmetry.

- Embedding of $\mathbb{Z}_{3}$ in a subgroup of $\mathrm{SU}(4)_{R}$ :

- $\quad$ into an $\mathrm{SU}(2)$ subgroup leads to $\mathcal{N}=2$ supersymmetric models with $\mathrm{SU}(2)_{R} R$-symmetry; and

- $\quad$ into an $\mathrm{SU}(3)$ subgroup leads to $\mathcal{N}=1$ supersymmetric models with $\mathrm{U}(1)_{R} R$-symmetry.

The preferred amount of supersymmetry is obtained by the last choice, therefore it is the one that is adopted [162]. A generator $g$ of $\mathbb{Z}_{3}$ can be labelled by three integers, as $\vec{a}=\left(a_{1}, a_{2}, a_{3}\right)$ [163] satisfying the following relation:

$$
a_{1}+a_{2}+a_{3}=0 \bmod 3 .
$$

As mentioned above, each kind of fields of the theory is accommodated in different representations of $\mathrm{SU}(4)_{R}$, therefore $\mathbb{Z}_{3}$ acts on the fields in a non-trivial way. Gauge fields and gauginos are singlets under $\mathrm{SU}(4)_{R}$, therefore the geometric action of the $\mathbb{Z}_{3}$ is trivial. The action on the three complex scalar fields is expressed by the matrix $\gamma(g)_{i j}=\delta_{i j} \omega^{a_{i}}$, where $\omega=e^{\frac{2 \pi}{3}}$, while on the fermions it is given by the matrix $\gamma(g)_{i j}=\delta_{i j} \omega^{b_{i}}$, where $b_{i}=-\frac{1}{2}\left(a_{i+1}+a_{i+2}-a_{i}\right)$ modulo 3. In this case, the generator, $g$, is given by the three integers $(1,1,-2)$, which means that $a_{i}=b_{i}$. Furthermore, the matter fields are not gauge invariant, thus $\mathbb{Z}_{3}$ acts also on their gauge indices. The action is given by the following matrix (These 
blocks may not have the same dimensionality (see e.g. [164-166]) but, in this case, they do, in order to result with a projected theory which is anomaly free.):

$$
\gamma_{3}=\left(\begin{array}{ccc}
\mathbf{1}_{N} & 0 & 0 \\
0 & \omega \mathbf{1}_{N} & 0 \\
0 & 0 & \omega^{2} \mathbf{1}_{N}
\end{array}\right) .
$$

The projection affects the spectrum of the resulting theory, in the following way. Every field that is not invariant under the action of $\mathbb{Z}_{3}$ on both the "geometric" (In the case of ordinary reduction of a 10-d $\mathcal{N}=1$ SYM theory, one obtains an $\mathcal{N}=4$ SYM Yang-Mills theory in four dimensions having a global $\mathrm{SU}(4)_{R}$ symmetry, which is identified as the tangent space $\mathrm{SO}(6)$ of the extra dimensions $\left.[33-35,167]\right)$ and gauge indices is filtered out and does not take part in the projected theory [163]. The projection for the gauge fields is $A_{\mu}=\gamma_{3} A_{\mu} \gamma_{3}^{-1}$. Therefore, taking into account Equation (45), the gauge group of the projected theory is the $\mathrm{H}=\mathrm{SU}(\mathrm{N}) \times \mathrm{SU}(\mathrm{N}) \times \mathrm{SU}(\mathrm{N})$. Regarding the scalar fields, they transform non-trivially under the gauge and $R$-symmetry, therefore the projection is $\phi_{I J}^{i}=\omega^{I-J+a_{i}} \phi_{I J}^{i}$, where $I, J$ are gauge indices. Thus, $J=I+a_{i}$, which means that the surviving scalars are of the form $\phi_{I, J+a_{i}}$ and belong to the following representation of the gauge group, $H$ :

$$
3 \cdot((N, \bar{N}, 1)+(\bar{N}, 1, N)+(1, N, \bar{N})) .
$$

The same holds for the fermions, therefore the projection is $\psi_{I J}^{i}=\omega^{I-J+b_{i}} \psi_{I J}^{i}$, which means that the surviving fermions have the form $\psi_{I, I+b_{i}}^{i}$, belonging to the same representation as the scalars, Equation (46), as expected since the remnant supersymmetry is $\mathcal{N}=1$. It is worth-noting that the representations in which scalars and fermions are encountered, Equation (46), are anomaly free. Therefore, it is understood that fermions fall into chiral representations of $\mathrm{H}$, which are divided into three generations, since the particle content consists of three $\mathcal{N}=1$ chiral supermultiplets.

The interactions between the fields of the projected theory are determined by the superpotential, the form of which is specified by the superpotential of the initial $\mathcal{N}=4$ SYM theory $[168,169]$ :

$$
W_{\mathcal{N}=4}=\epsilon_{i j k} \operatorname{Tr}\left(\Phi^{i} \Phi^{j} \Phi^{k}\right)
$$

where $\Phi^{i}, \Phi^{j}, \Phi^{k}$ are the three chiral superfields of the theory, before the projection. The orbifold projection does not affect the structure of the superpotential, however it includes the interactions among the fields that passed through the filtering of the orbifold projection to the $\mathcal{N}=1$ theory:

$$
W_{\mathcal{N}=1}^{(p r o j)}=\sum_{I} \epsilon_{i j k} \Phi_{I, I+a_{i}}^{i} \Phi_{I+a_{i} I+a_{i}+a_{j}}^{j} \Phi_{I+a_{i}+a_{j}, I}^{k}
$$

\subsection{Dynamical Generation of Twisted Fuzzy Spheres}

The corresponding scalar potential is originating from the superpotential $W_{\mathcal{N}=1}^{p r o j}$, Equation (48), as:

$$
V_{\mathcal{N}=1}^{p r o j}(\phi)=\frac{1}{4} \operatorname{Tr}\left(\left[\phi^{i}, \phi^{j}\right]^{\dagger}\left[\phi^{i}, \phi^{j}\right]\right),
$$

where $\phi^{i}$ is the scalar part of the superfield, $\Phi^{i}$. Aiming at vacua which could admit an interpretation of fuzzy extra dimensions, the potential $V_{\mathcal{N}=1}^{p r o j}(\phi)$ has to be modified, since it gets minimized by vanishing vevs of the fields. Therefore, to result with non-vanishing minima of the scalar potential, $V_{\mathcal{N}=1}^{p r o j}(\phi), \mathcal{N}=1$ soft supersymmetric terms of the following form have to be introduced (For the current purpose, the SSB terms that are introduced in $V_{\mathcal{N}=1}^{\text {proj }}(\phi)$, are purely scalar. However, more SSB terms have to be included to obtain the complete SSB sector [170].): 


$$
V_{S S B}=\frac{1}{2} \sum_{i} m_{i}^{2} \phi^{i+} \phi^{i}+\frac{1}{2} \sum_{i, j, k} h_{i j k} \phi^{i} \phi^{j} \phi^{k}+\text { h.c. }
$$

with $h_{i j k}=0$, unless $i+j+k \equiv 0 \bmod 3$. In addition, the $D$-terms contributing to the scalar potential are:

$$
V_{D}=\frac{1}{2} D^{2}=\frac{1}{2} D^{I} D_{I}
$$

where $D^{I}=\phi_{i}^{\dagger} T^{I} \phi^{i}$ and $T^{I}$ are the generators of the gauge group in the representation of the corresponding chiral multiplet. The total potential of the theory is given as a combination of all the above contributions:

$$
V=V_{\mathcal{N}=1}^{p r o j}+V_{S S B}+V_{D}
$$

Fixing appropriately the parameters $m_{i}^{2}$ and $h_{i j k}$ of Equation (50) as $m_{i}^{2}=1$ and $h_{i j k}=\epsilon_{i j k}$, the scalar potential, Equation (52), can now be written as:

$$
V=\frac{1}{4}\left(F^{i j}\right)^{\dagger} F^{i j}+V_{D}
$$

where the tensor $F^{i j}$ introduced above is defined as:

$$
F^{i j}=\left[\phi^{i}, \phi^{j}\right]-i \epsilon^{i j k}\left(\phi^{k}\right)^{\dagger}
$$

The first term in the above expression of the potential, Equation (53), is positive, thus the global minimum of the potential is:

$$
\left[\phi^{i}, \phi^{j}\right]=i \epsilon_{i j k}\left(\phi^{k}\right)^{\dagger}, \quad \phi^{i}\left(\phi^{j}\right)^{\dagger}=R^{2},
$$

where $\left(\phi^{i}\right)^{\dagger}$ is the Hermitian conjugate of $\phi^{i}$ and $\left[R^{2}, \phi^{i}\right]=0$. Now, it has become manifest that the above relations are associated to a fuzzy sphere. This gets clearer if the untwisted fields, $\widetilde{\phi}^{i}$, are introduced, defined by:

$$
\phi^{i}=\Omega \widetilde{\phi}^{i}
$$

where $\Omega \neq 1$ satisfies the following relations:

$$
\Omega^{3}=1, \quad\left[\Omega, \phi^{i}\right]=0, \quad \Omega^{\dagger}=\Omega^{-1}, \quad\left(\widetilde{\phi}^{i}\right)^{\dagger}=\widetilde{\phi}^{i} \Leftrightarrow\left(\phi^{i}\right)^{\dagger}=\Omega \phi^{i} .
$$

Therefore, the fuzzy sphere relations are recovered, Equation (55), generated by $\widetilde{\phi}^{i}$ :

$$
\left[\widetilde{\phi}^{i}, \widetilde{\phi}^{j}\right]=i \epsilon_{i j k} \widetilde{\phi}^{k}, \quad \widetilde{\phi}^{i} \widetilde{\phi}^{i}=R^{2},
$$

designating that the noncommutative space, dynamically generated by $\phi^{i}$, is in fact a twisted fuzzy sphere, $\widetilde{S}_{N}^{2}$. Specific configurations of the twisted fields, $\phi^{i}$, i.e., fields satisfying Equation (55), can be obtained:

$$
\phi^{i}=\Omega\left(\mathbf{1}_{3} \otimes \lambda_{(N)}^{i}\right)
$$

where $\lambda_{(N)}^{i}$ are the three $\mathrm{SU}(2)$ generators in the $\mathrm{N}$-dimensional irreducible representation and $\Omega$ is the matrix:

$$
\Omega=\Omega_{3} \otimes \mathbf{1}_{N}, \quad \Omega_{3}=\left(\begin{array}{lll}
0 & 1 & 0 \\
0 & 0 & 1 \\
1 & 0 & 0
\end{array}\right), \quad \Omega^{3}=\mathbf{1} .
$$


The transformation in Equation (56) implies that the "off-diagonal" orbifold sectors, Equation (46), now take the following block-diagonal form:

$$
\phi^{i}=\left(\begin{array}{ccc}
0 & \left(\lambda_{(N)}^{i}\right)_{(N, \bar{N}, 1)} & 0 \\
0 & 0 & \left(\lambda_{(N)}^{i}\right)_{(1, N, \bar{N})} \\
\left(\lambda_{(N)}^{i}\right)_{(\bar{N}, 1, N)} & 0 & 0
\end{array}\right)=\Omega\left(\begin{array}{ccc}
\lambda_{(N)}^{i} & 0 & 0 \\
0 & \lambda_{(N)}^{i} & 0 \\
0 & 0 & \lambda_{(N)}^{i}
\end{array}\right) .
$$

The untwisted fields, $\widetilde{\phi}^{i}$, are written in a block-diagonal form, with each block being identified as a fuzzy sphere, since the corresponding commutation relation, Equation (58), is satisfied for each one separately. The above configuration, Equation (61), determines the vacuum of the theory and consists of three fuzzy spheres of the same radii, with relative angles $2 \pi / 3$ (with $\Omega$ expressed in the representation of $\gamma_{3}$ in Equation (45)). In other words, the expression of $\phi^{i}$ can be interpreted as the twisted equivalent of three fuzzy spheres. It is notable that $F^{i j}$, Equation (54), is identified as the field strength tensor of the dynamically generated fuzzy extra dimensions. In addition, the term related to the $D$-terms, $V_{D}$, of the total potential expression is responsible for a change on the radius of the sphere $[70,71,73,171]$.

\subsection{Chiral Models after the Fuzzy Orbifold Projection-The $S U(3)_{c} \times S U(3)_{L} \times S U(3)_{R}$ Model}

The orbifold projection breaks the gauge group of the initial SYM theory, $\mathrm{SU}(3 \mathrm{~N})$, to a subgroup. Depending on the way the breaking takes place, it affects the resulting gauge symmetry. The minimal, anomaly free cases of resulting unified gauge theories are described by the groups, $\mathrm{SU}(4) \times \mathrm{SU}(2) \times \mathrm{SU}(2), \mathrm{SU}(4)^{3}$ and $\mathrm{SU}(3)^{3}$ (For a similar approach in the Yang-Mills matrix models framework see $[172,173]$.). The case of interest is the trinification group $\mathrm{SU}(3)_{c} \times \mathrm{SU}(3)_{L} \times \mathrm{SU}(3)_{R}$ $[174,175]$ (see also $[159,160,176-180]$ for a string theory approach). First, the integer $\mathrm{N}$ is written as $N=n+3$, therefore for each $\mathrm{SU}(\mathrm{N})$, the embedding would be:

$$
\operatorname{SU}(N) \supset \operatorname{SU}(n) \times S U(3) \times U(1) .
$$

Therefore, the embedding for the total gauge group $\mathrm{SU}(\mathrm{N})^{3}$ would be the following:

$$
S U(N)^{3} \supset S U(n) \times S U(3) \times S U(n) \times S U(3) \times S U(n) \times S U(3) \times U(1)^{3} .
$$

The U(1) factors produced are not taken into account (Due to anomalous gaining mass by the Green-Schwarz mechanism and thus they decouple at the low energy regime [165].) and the representations are decomposed according to the above relation, Equation (63), as:

$$
\begin{aligned}
& S U(n) \times S U(n) \times S U(n) \times S U(3) \times S U(3) \times S U(3), \\
& (n, \bar{n}, 1 ; 1,1,1)+(1, n, \bar{n} ; 1,1,1)+(\bar{n}, 1, n ; 1,1,1)+(1,1,1 ; 3, \overline{3}, 1) \\
& +(1,1,1 ; 1,3, \overline{3})+(1,1,1 ; \overline{3}, 1,3)+(n, 1,1 ; 1, \overline{3}, 1)+(1, n, 1 ; 1,1, \overline{3}) \\
& +(1,1, n ; \overline{3}, 1,1)+(\bar{n}, 1,1 ; 1,1,3)+(1, \bar{n}, 1 ; 3,1,1)+(1,1, \bar{n} ; 1,3,1) .
\end{aligned}
$$

Taking into consideration the decomposition of $\mathrm{SU}(\mathrm{N})^{3}$, Equation (62), the gauge group breaks down to $\mathrm{SU}(3)^{3}$. Now, under $\mathrm{SU}(3)^{3}$, the surviving fields of the resulting theory transform as:

$$
\begin{gathered}
\operatorname{SU}(3) \times \operatorname{SU}(3) \times \operatorname{SU}(3), \\
((3, \overline{3}, 1)+(\overline{3}, 1,3)+(1,3, \overline{3})),
\end{gathered}
$$

which is the corresponding chiral representation of the trinification group, under which the quarks and leptons of the first family transform as: 


$$
q=\left(\begin{array}{lll}
d & u & h \\
d & u & h \\
d & u & h
\end{array}\right) \sim(3, \overline{3}, 1), q^{c}=\left(\begin{array}{lll}
d^{c} & d^{c} & d^{c} \\
u^{c} & u^{c} & u^{c} \\
h^{c} & h^{c} & h^{c}
\end{array}\right) \sim(\overline{3}, 1,3), \lambda=\left(\begin{array}{ccc}
N & E^{c} & \mathrm{v} \\
E & N^{c} & e \\
v^{c} & e^{c} & S
\end{array}\right) \sim(1,3, \overline{3}),
$$

respectively. The matrices of the other two families are obtained in a similar way (It is worth-noting that the resulting theory can be promoted to a finite theory up to two loops (see [176,181-183]) and give testable predictions $[176,184,185]$, too.). In addition, fuzzy orbifolds can also be used to break the gauge group of the unified theory down to MSSM and then to the $\mathrm{SU}(3)_{c} \times \mathrm{U}(1)_{\mathrm{em}}$.

A reasonable extension of the above concept and methodology in the context of Matrix Models (MM) has been studied in [186] (see also [187]). The MMs were first introduced by Banks-Fischler-Shenker-Susskind (BFSS) and Ishibashi-Kawai-Kitazawa-Tsuchiya (IKKT) and provide a non-perturbative approach of M-theory and type IIB string theory, respectively [58,102]. In addition, MMs can also be employed for model building at low energies, producing various interesting solutions on strings, D-branes [58,188-190] and noncommutative/fuzzy spaces, such as fuzzy tori and spheres [191-193]. Since such constructions lead to non-Abelian gauge theories, it is reasonable to explore the possibility of building phenomenologically viable particle physics models. Moreover, a MM involving orbifold projection was proposed by Aoki-Iso-Suyama (AIS) [194], as a specific projection of the IKKT model, being related to the above construction, in which fuzzy extra dimensions are dynamically generated [74,75]. Application of a $\mathbb{Z}_{3}$ orbifold projection, reduces the initial symmetry of the IKKT matrix model with matrix size $3 \mathrm{~N} \times 3 \mathrm{~N}$, from $\mathrm{SO}(9,1) \times \mathrm{U}(3 \mathrm{~N})$ to $\mathrm{SO}(3,1) \times \mathrm{U}(\mathrm{N})^{3}$. This model is $4-\mathrm{d}, \mathcal{N}=1$ supersymmetric, chiral of Yang-Mills type. The fermions that are invariant under the $\mathbb{Z}_{3}$ projection, transform in the bifundamental representations under the initial, unbroken gauge symmetry exactly as in the construction described above. Future plans involve the extension of the project started in $[186,187]$ in the context of IKKT models making use of an orbifold projection.

\section{Gravity as a Gauge Theory}

In this section, we recall the interpretation of various gravity theories as gauge theories [79-91], to translate it later into the framework of noncommutative geometry.

\subsection{4-d Einstein's Gravity as a Gauge Theory}

For over one hundred years, General Relativity has been considered a very solid and successful theory, having passed various tests throughout these years. It is formulated in a geometric way, in difference to the rest of the interactions, which are described as gauge theories. To make contact between gravitational and the other interactions, in the mid 1950s [79], an undertaking began in this direction, i.e., the gauge-theoretic approach of gravity [80-85]. Let us recall the basic features of this approach for the 4-d Einstein's gravity.

To aim at a gauge-theoretic approach of 4-d gravity, the vierbein formulation of General Relativity has to be taken into account. Depending on the presence or not of the cosmological constant, the gauge theory is constructed on the 4-d Minkowski, de Sitter or anti-de Sitter spacetime, $M^{4}, d S_{4}, A d S_{4}$, with gauge groups the Poincaré, de Sitter or anti-de Sitter groups, $\mathrm{ISO}(1,3), \mathrm{SO}(1,3)$, and $\mathrm{SO}(2,2)$, respectively. The choice of these groups as the symmetry groups is reasonable, since they are the isometry groups of corresponding spacetimes. Let us start with the case in which there is no cosmological constant included, i.e., the case of the Poincaré group. The corresponding algebra is determined by the following commutation relations, which its generators satisfy:

$$
\left[M_{a b}, M_{c d}\right]=4 \eta_{[a[c} M_{d] b]}, \quad\left[P_{a}, M_{b c}\right]=2 \eta_{a[b} P_{c]}, \quad\left[P_{a}, P_{b}\right]=0,
$$

where $\eta_{a b}=\operatorname{diag}(-1,1,1,1)$ is the Minkowski metric, $M_{a b}$ are the generators of the Lorentz group (Lorentz transformations) and $P_{a}$ are the generators of the local translations. According to the standard 
gauging procedure, the gauge potential, $A_{\mu}$, is introduced and it is expressed as a decomposition on the generators of the Poincaré algebra, as follows:

$$
A_{\mu}(x)=e_{\mu}{ }^{a}(x) P_{a}+\frac{1}{2} \omega_{\mu}^{a b}(x) M_{a b} .
$$

The functions attached to the generators are the gauge fields of the theory and, in this case, they are identified as the vierbein, $e_{\mu}{ }^{a}$, and the spin connection, $\omega_{\mu}{ }^{a b}$, which correspond to the translations, $P_{a}$, and Lorentz generators, $M_{a b}$, respectively. This way, considering the vierbein as gauge field, the mixing between the internal symmetry and spacetime is achieved and that is what makes this kind of construction special, compared to the gauge theories describing other interactions. The connection transforms according to the following rule:

$$
\delta A_{\mu}=\partial_{\mu} \epsilon+\left[A_{\mu}, \epsilon\right],
$$

where $\epsilon=\epsilon(x)$ is the parameter of the gauge transformation that can also be expanded on the various generators of the algebra:

$$
\epsilon(x)=\xi^{a}(x) P_{a}+\frac{1}{2} \lambda^{a b}(x) M_{a b} .
$$

The transformations of the gauge fields are obtained after putting together Equations (69) and (71) with Equation (70). The resulting expressions for the transformations are:

$$
\begin{gathered}
\delta e_{\mu}{ }^{a}=\partial_{\mu} \xi^{a}+\omega_{\mu}{ }^{a b} \xi_{b}-\lambda^{a}{ }_{b} e_{\mu}{ }^{b}, \\
\delta \omega_{\mu}{ }^{a b}=\partial_{\mu} \lambda^{a b}-2 \lambda^{[a}{ }_{c} \omega_{\mu}{ }^{c b]} .
\end{gathered}
$$

According to the standard procedure followed in gauge theories, the corresponding field strength tensor of the gauge theory is defined:

$$
R_{\mu v}(A)=2 \partial_{[\mu} A_{v]}+\left[A_{\mu}, A_{v}\right]
$$

and also becomes expanded on the generators:

$$
R_{\mu v}(A)=R_{\mu v}{ }^{a}(e) P_{a}+\frac{1}{2} R_{\mu \nu}{ }^{a b}(\omega) M_{a b},
$$

where $R_{\mu \nu}{ }^{a}(e)$ and $R_{\mu \nu}{ }^{a b}(\omega)$ are the curvatures of the gauge fields, identified as the torsion and curvature, respectively. Their expressions are obtained by replacing Equations (69) and (75) into Equation (74):

$$
\begin{gathered}
R_{\mu \nu}{ }^{a}(e)=2 \partial_{[\mu} e_{v]}^{a}-2 \omega_{[\mu}^{a b} e_{\nu] b}, \\
R_{\mu \nu}{ }^{a b}(\omega)=2 \partial_{[\mu} \omega_{\nu]}^{a b}-2 \omega_{\left[\mu^{a c}\right.} \omega_{\nu] c}^{b} .
\end{gathered}
$$

Concerning the dynamic part of the theory, the obvious choice is an action of Yang-Mills type, which is invariant under the Poincare group. However, intenting to result with the Einstein-Hilbert action, the Poincaré symmetry of the action has to be broken to the Lorentz. This can be achieved by the employment of a spontaneous symmetry breaking, induced by a scalar field which belongs to the fundamental representation of the $\mathrm{SO}(1,4)[81,83,84]$. The choice of the de Sitter group is equivalent to that of the Poincare group, and in this case it is preferred, because all generators can be considered on equal footing. The translational part of the theory is broken by the spontaneous symmetry breaking, which leads to a constrained theory with vanishing torsion and an action involving the Ricci scalar, respecting only the Lorentz symmetry, i.e., Einstein-Hilbert action.

Concluding, Einstein's four-dimensional gravity can be formulated as a gauge theory of the Poincaré group, as far as the kinematic part is concerned, i.e., the transformation of the fields and the 
expressions of the curvature tensors. When it comes to the dynamic part, instead of the Poincare group, the de Sitter one is the symmetry which the initial Yang-Mills action respects. In turn, the inclusion of a scalar field and the addition of an appropriate potential term in the Lagrangian leads to a spontaneous symmetry breaking, leading to the Einstein-Hilbert action.

An alternative option to result with an action with Lorentz symmetry is to claim that the action has to be invariant under the Lorentz symmetry and not under the total Poincare with which one begins. This means that the curvature tensor corresponding to the translations has to be set to zero, deriving the torsionless condition. The torsionless condition is the constraint that is necessary for the capture of the Lorentz symmetry. Solution of this constraint leads to an expression relating the spin connection with the vielbein:

$$
\omega_{\mu}^{a b}=\frac{1}{2} e^{v a}\left(\partial_{\mu} e_{\nu}^{b}-\partial_{\nu} e_{\mu}^{b}\right)-\frac{1}{2} e^{\nu b}\left(\partial_{\mu} e_{\nu}{ }^{a}-\partial_{\nu} e_{\mu}^{a}\right)-\frac{1}{2} e^{\rho a} e^{\sigma b}\left(\partial_{\rho} e_{\sigma c}-\partial_{\sigma} e_{\rho c}\right) e_{\mu}^{c} .
$$

However, after the imposition of the constraint, straightforward consideration of an action of Yang-Mills type respecting the (remnant) Lorentz symmetry would lead to an $R(M)^{2}$ action, which is not the correct one, since the target is the reproduction of the results of general relativity. Such an action would imply the presence of a dimensionless coupling constant of gravity, but that is not the case. To result with the Einstein-Hilbert action, which involves a dimensionful coupling constant, the action has to be considered in a non-straightforward way, specifically, building Lorentz invariants out of the quantities (curvature tensors) of the theory. The one that is constructed under certain contractions of the curvature tensor is the correct one, ensuring the correct dimensionality of the coupling constant. The latter is eventually identified as the Ricci scalar and the corresponding action is the Einstein-Hilbert action.

\subsection{3-d Gravity as a Gauge Theory}

The construction of a gauge theory describing the 3-d Einstein's gravity (without cosmological constant) is less tedious than the above 4-d case, since it can be completely recovered considering the 3-d Poincare group as the gauge group, for both the kinematic and the dynamic parts [91]. The 3-d Einstein-Hilbert action is written as:

$$
S_{\mathrm{EH} 3}=\frac{1}{2} \int d^{3} x \epsilon^{\mu \nu \rho} \epsilon_{a b c} e_{\mu}{ }^{a} R_{v \rho}^{b c}(\omega),
$$

which, as shown in [91], is identical to a gauge theory of Chern-Simons type of ISO(1,2). This algebra comprises six generators, three local translations, $P_{a}$ and the three Lorentz transformations, $M^{a}=\epsilon^{a b c} M_{b c}$, with $a=1,2,3$, which satisfy the following commutation relations:

$$
\left[M_{a}, M_{b}\right]=\epsilon_{a b c} M^{c}, \quad\left[P_{a}, M_{b}\right]=\epsilon_{a b c} P^{c}, \quad\left[P_{a}, P_{b}\right]=0 .
$$

Following the same gauging procedure as in the 4-d case, the gauge connection and gauge parameter are written as:

$$
A_{\mu}=e_{\mu}{ }^{a} P_{a}+\omega_{\mu}{ }^{a} M_{a}, \quad \epsilon=\xi^{a} P_{a}+\lambda^{a} M_{a} .
$$

The above considerations are sufficient to calculate the gauge transformations of the fields and the component curvature tensors. Appropriate choice of the quadratic form of the algebra leads to a Chern-Simons action that is identical to the Einstein-Hilbert action, Equation (79). In addition, 3-d gravitational theory is also retrieved by a gauge-theoretic approach in the case that a cosmological constant is included. For this purpose, the gauge groups considered are the 3-d dS or AdS groups, $\mathrm{SO}(1,3)$ and $\mathrm{SO}(2,2)$, respectively. In this case, the generators of the local translations do not commute, satisfying the following commutation relation:

$$
\left[P_{a}, P_{b}\right]=\lambda M_{a b}
$$


where $\lambda$ is the cosmological constant. The rest of the procedure remains the same as the one without the cosmological constant.

\subsection{4-d Weyl Gravity as a Gauge Theory}

Besides Einstein's gravity, also Weyl's gravity can be successfully described as a gauge theory of the 4-d conformal group, $\mathrm{SO}(2,4)$. In this case, the transformations of the fields and the expressions of the curvature tensors are determined in a straightforward way. The initial action that is considered is an $\mathrm{SO}(2,4)$ invariant action of Yang-Mills type and is broken by imposition of specific conditions (constraints) on the curvature tensors. After taking into consideration the constraints, the resulting action of the theory is the Weyl action [86-88] (see also [89,90]).

The generators of the conformal algebra of $\mathrm{SO}(2,4)$ are the local translations $\left(P_{a}\right)$, the Lorentz transformations $\left(M_{a b}\right)$, the conformal boosts $\left(K_{a}\right)$ and the dilatations $(D)$. The algebra is determined by their commutation relations:

$$
\begin{gathered}
{\left[M_{a b}, M^{c d}\right]=4 M_{[a}^{[d} \delta_{b]^{c}}^{c]} \quad\left[M_{a b}, P_{c}\right]=2 P_{[a} \delta_{b] c}, \quad\left[M_{a b}, K_{c}\right]=2 K_{[a} \delta_{b] c},} \\
{\left[P_{a}, D\right]=P_{a}, \quad\left[K_{a}, D\right]=-K_{a}, \quad\left[P_{a}, K_{b}\right]=2\left(\delta_{a b} D-M_{a b}\right),}
\end{gathered}
$$

where $a, b, c, d=1 \ldots 4$. Now, according to the gauging procedure, the gauge potential of the theory has to be determined, i.e.

$$
A_{\mu}=e_{\mu}^{a} P_{a}+\frac{1}{2} \omega_{\mu}^{a b} M_{a b}+b_{\mu} D+f_{\mu}^{a} K_{a}
$$

in which a gauge field has been associated with each generator. The gauge connection, Equation (84), transforms according to the following rule:

$$
\delta_{\epsilon} A_{\mu}=D_{\mu} \epsilon=\partial_{\mu} \epsilon+\left[A_{\mu}, \epsilon\right]
$$

where $\epsilon$ is a gauge parameter which takes values in the Lie algebra of the $\mathrm{SO}(2,4)$ group and therefore it can be written as:

$$
\epsilon=\epsilon_{P}^{a} P_{a}+\frac{1}{2} \epsilon_{M}^{a b} M_{a b}+\epsilon_{D} D+\epsilon_{K}^{a} K_{a} .
$$

Combining Equations (84)-(86), the transformations of the gauge fields of the theory are obtained:

$$
\begin{aligned}
\delta e_{\mu}{ }^{a} & =\partial_{\mu} \epsilon_{P}^{a}+2 i e_{\mu b} \epsilon_{M}^{a b}-i \omega_{\mu}^{a b} \epsilon_{P b}-b_{\mu} \epsilon_{K}^{a}+f_{\mu}{ }^{a} \epsilon_{D}, \\
\delta \omega_{\mu}{ }^{a b} & =\frac{1}{2} \partial_{\mu} \epsilon_{M}^{a b}+4 i e_{\mu}{ }^{a} \epsilon_{P}{ }^{b}+\frac{i}{4} \omega_{\mu}{ }^{a c} \epsilon_{M c}{ }^{b}+i f_{\mu}{ }^{a} \epsilon_{K}{ }^{b}, \\
\delta b_{\mu} & =\partial_{\mu} \epsilon_{D}-e_{\mu}{ }^{a} \epsilon_{K a}+f_{\mu}{ }^{a} \epsilon_{P a} \\
\delta f_{\mu}{ }^{a} & =\partial_{\mu} \epsilon_{K}{ }^{a}+4 i e_{\mu}{ }^{a} \epsilon_{D}-i \omega_{\mu}{ }^{a b} \epsilon_{K b}-4 i b_{\mu} \epsilon_{P}{ }^{a}+i f_{\mu}{ }^{b} \epsilon_{M b}^{a} .
\end{aligned}
$$

Now, employing the definition of the field strength tensor for non-Abelian gauge theories:

$$
R_{\mu v}=2 \partial_{[\mu} A_{v]}-i\left[A_{\mu}, A_{v}\right]
$$

the expressions of the component curvature tensors are obtained:

$$
\begin{aligned}
R_{\mu v}^{a}(P) & =2 \partial_{[\mu} e_{v]}^{a}+f_{[\mu}^{a} b_{v]}+e_{[\mu}^{b} \omega_{v]}^{a c} \delta_{b c}, \\
R_{\mu \nu}^{a b}(M) & =\partial_{[\mu} \omega_{v]}^{a b}+\omega_{[\mu}^{c a} \omega_{v]}^{d b} \delta_{c d}+e_{[\mu}^{a} e_{v]}^{b}+f_{[\mu}^{a} f_{v]}^{b}, \\
R_{\mu v}(D) & =2 \partial_{[\mu} b_{v]}+f_{[\mu}^{a} e_{v]}^{b} \delta_{a b}, \\
R_{\mu \nu}^{a}(K) & =2 \partial_{[\mu} f_{v]}^{a}+e_{[\mu}^{a} b_{v]}+f_{[\mu}^{b} \omega_{v]}^{a c} \delta_{b c} .
\end{aligned}
$$


Now, regarding the action, it is taken to be an $\mathrm{SO}(2,4)$ invariant Yang-Mills action. Imposition of specific constraints break the initial symmetry [86-88], specifically, the torsionless condition, $R(P)=0$, along with an additional constraint on $R(M)$. The algebraic solution of these constraints leads to expressions of the fields $\omega_{\mu}^{a b}$ and $f_{\mu}{ }^{a}$ in terms of the independent fields $e_{\mu}{ }^{a}$ and $b_{\mu}$. Finally, $b_{\mu}$ can be gauge fixed to $b_{\mu}=0$ and, therefore, the resulting action is the well-known Weyl action, being diffeomorphism and scale invariant.

Besides the above, well-established way of breaking the initial symmetry to a symmetry of scale and diffeomorphism invariance, we suggest another way of symmetry breaking, this time leading to the Lorentz symmetry. The transformations of the fields and component tensor expressions determined above, along with this alternative symmetry breaking become useful below, serving as the commutative limit of the noncommutative 4-d gravity model we present. This alternative symmetry breaking could take place including two scalar fields in the fundamental representation of the $\mathrm{SO}(2,4)$ gauge group [195]. This is a rather natural generalization of the way the 4-d de Sitter group was broken down to the Lorentz by a scalar in the fundamental of the corresponding group, $\mathrm{SO}(1,4)$, as explained above in the case of the 4-d Einstein gravity, in Section 6.1. The two scalars could be responsible for triggering a spontaneous symmetry breaking in a more complete theory in which matter fields would be included. The resulting action of the resulting $4-\mathrm{d}$ theory should respect Lorentz symmetry.

\section{3-d Gravity as a Gauge Theory on Noncommutative Spaces}

In Section 6.2, we recal that 3-d gravity can be described as a Chern-Simons gauge theory. At the same time, there exists a solid framework for constructing noncommutative gauge theories, as briefly reviewed in Section 3. Therefore, it seems reasonable to combine the above two and construct a 3-d gravity model as a noncommutative gauge theory. The first step towards this direction is the identification of a suitable noncommutative space that will accommodate the gauge theory $[123,124,196]$.

An appropriate 3-d fuzzy space is constructed as a foliation of the 3-d Euclidean space by multiple fuzzy spheres of different radii, called $\mathbb{R}_{\lambda}^{3}$, first considered in [125] (see also [126]). In this space, the noncommutative coordinates satisfy the commutation relation of the $\mathrm{SU}(2)$ algebra, but, unlike the case of the fuzzy sphere, the matrices are not considered to be proportional to the generators of $\mathrm{SU}(2)$ in an irreducible higher-dimensional representation, but in a reducible one. Consideration of a reducible representation means that the coordinates are given as block-diagonal matrices, with each block being an irreducible representation, i.e., a fuzzy sphere. Thus, the Hilbert space is:

$$
\mathcal{H}=\oplus[\ell], \quad \ell=0,1 / 2,1, \ldots
$$

The three operators that are identified as the coordinates satisfy the following commutation relation:

$$
\left[X_{i}, X_{j}\right]=i \lambda \epsilon_{i j k} X_{k}
$$

and are described by matrices in reducible representations of $s u(2)$ (cf. [125]). In other words, allowing $X_{i}$ to live in a reducible representation is equivalent to considering a sum of fuzzy two-spheres of different radii. Therefore, the whole space can be viewed as a discrete foliation of 3-d Euclidean space by fuzzy two-spheres, each being a leaf of the foliation (In the Lorentzian case, there exists a similar construction, i.e., the foliation of the 3-d Minkowski space by fuzzy hyperboloids [128].) (cf. [197]).

The above space, $\mathbb{R}_{\lambda}^{3}$, admits an $\mathrm{SO}(4)$ symmetry [127], which is the one that is gauged. In this procedure, the generic problem of non-Abeilian noncommutative gauge theories is encountered, i.e., the non-closure of the anti-commutators of the generators of the algebra. The indicated treatment for this issue is the specification of the representation in which generators are accommodated and minimally extend the algebra with the necessary operators that are produced by the anti-commutators. Accordingly, the gauge theory considered in this case is the $\mathrm{U}(2) \times \mathrm{U}(2)$ in a fixed representation, because of the inclusion of the operators $\mathbf{1}$ and $\gamma_{5}$. 
Now, having determined the fuzzy space and the gauge group, the procedure followed is similar to the one of the continuous case, modified for the noncommutative framework. First, the commutation and anti-commutation relations of the generators of the gauge group are obtained:

$$
\begin{gathered}
{\left[P_{a}, P_{b}\right]=i \epsilon_{a b c} M_{c}, \quad\left[P_{a}, M_{b}\right]=i \epsilon_{a b c} P_{c}, \quad\left[M_{a}, M_{b}\right]=i \epsilon_{a b c} M_{c},} \\
\left\{P_{a}, P_{b}\right\}=\frac{1}{2} \delta_{a b} \mathbf{1}, \quad\left\{P_{a}, M_{b}\right\}=\frac{1}{2} \delta_{a b} \gamma_{5}, \quad\left\{M_{a}, M_{b}\right\}=\frac{1}{2} \delta_{a b} \mathbf{1} .
\end{gathered}
$$

Next, a gauge field has to be introduced for each generator writing down the gauge connection, which is used in promoting the coordinate to its covariant version:

$$
X_{\mu}=X_{\mu} \otimes i \mathbf{1}+e_{\mu}^{a} \otimes P_{a}+\omega_{\mu}^{a} \otimes M_{a}+A_{\mu} \otimes i \mathbf{1}+\widetilde{A}_{\mu} \otimes \gamma_{5},
$$

In addition, a gauge parameter is introduced and, since it is valued in the Lie algebra, it is expanded on its generators as:

$$
\epsilon=\xi^{a} \otimes P_{a}+\lambda^{a} \otimes M_{a}+\epsilon_{0} \otimes i \mathbf{1}+\widetilde{\epsilon}_{0} \otimes \gamma_{5} .
$$

Using the above relations and the transformation rule of the covariant coordinate, the transformations of the gauge fields are obtained:

$$
\begin{aligned}
\delta e_{\mu}^{a} & =-i\left[X_{\mu}+A_{\mu}, \xi^{a}\right]+2\left\{\omega_{\mu b}, \xi_{c}\right\} \epsilon^{a b c}+2\left\{e_{\mu b}, \lambda^{c}\right\} \epsilon^{a b c}+2 i\left[\lambda_{a}, \widetilde{A}_{\mu}\right]+2 i\left[\widetilde{\epsilon}_{0}, \omega_{\mu a}\right]+i\left[\epsilon_{0}, e_{\mu a}\right], \\
\delta \omega_{\mu}^{a} & =-i\left[X_{\mu}+A_{\mu}, \lambda^{a}\right]+2\left\{\omega_{\mu b}, \lambda_{c}\right\} \epsilon^{a b c}-\frac{1}{2}\left\{e_{\mu b}, \xi_{c}\right\} \epsilon^{a b c}+\frac{i}{2}\left[\xi^{a}, \widetilde{A}_{\mu}\right]+i\left[\epsilon_{0}, \omega_{\mu}^{a}\right]+\frac{i}{2}\left[\widetilde{\epsilon}_{0}, e_{\mu}^{a}\right], \\
\delta A_{\mu} & =-i\left[X_{\mu}+A_{\mu}, \epsilon_{0}\right]-i\left[\xi^{a}, e_{\mu a}\right]+4 i\left[\lambda^{a}, \omega_{\mu a}\right]-i\left[\widetilde{\epsilon}_{0}, \widetilde{A}_{\mu}\right], \\
\delta \widetilde{A}_{\mu} & =-i\left[X_{\mu}+A_{\mu}, \widetilde{\epsilon}_{0}\right]+2 i\left[\xi^{a}, \omega_{\mu a}\right]+2 i\left[\lambda^{a}, e_{\mu a}\right]+i\left[\epsilon_{0}, \widetilde{A}_{\mu}\right] .
\end{aligned}
$$

In addition, using the expressions of the covariant coordinate in the following relation:

$$
\mathcal{R}_{\mu v}=\left[\mathcal{X}_{\mu}, \mathcal{X}_{v}\right]-i \lambda \epsilon_{\mu \nu}{ }^{\rho} \mathcal{X}_{\rho},
$$

the corresponding curvatures are obtained, as well:

$$
\begin{aligned}
& T_{\mu \nu}^{a}=i\left[X_{\mu}+A_{\mu}, e_{v}^{a}\right]-i\left[X_{v}+A_{v}, e_{\mu}^{a}\right]-2 \epsilon^{a b c}\left(\left\{e_{\mu b}, \omega_{v c}\right\}+\left\{\omega_{\mu b}, e_{v c}\right\}\right) \\
& +2 i\left(\left[\omega_{\mu}{ }^{a}, \widetilde{A}_{v}\right]-\left[\omega_{v}{ }^{a}, \widetilde{A}_{\mu}\right]\right)-i \lambda \epsilon_{\mu \nu}^{\rho} e_{\rho}^{a} \text {, } \\
& R_{\mu v}^{a}=i\left[X_{\mu}+A_{\mu}, \omega_{v}^{a}\right]-i\left[X_{v}+A_{v}, \omega_{\mu}^{a}\right]+\epsilon^{a b c}\left(\frac{1}{2}\left\{e_{\mu b}, e_{v c}\right\}-2\left\{\omega_{\mu b}, \omega_{v c}\right\}\right) \\
& +\frac{i}{2}\left(\left[e_{\mu}^{a}, \widetilde{A}_{v}\right]-\left[e_{v}^{a}, \widetilde{A}_{\mu}\right]\right)-i \lambda \epsilon_{\mu \nu}^{\rho} \omega_{\rho}^{a}, \\
& F_{\mu \nu}=i\left[X_{\mu}+A_{\mu}, X_{v}+A_{v}\right]-i\left[e_{\mu}{ }^{a}, e_{v a}\right]+4 i\left[\omega_{\mu}{ }^{a}, \omega_{v a}\right]-i\left[\widetilde{A}_{\mu}, \widetilde{A}_{v}\right]-i \lambda \epsilon_{\mu \nu}{ }^{\rho}\left(X_{\rho}+A_{\rho}\right) \text {, } \\
& \widetilde{F}_{\mu v}=i\left[X_{\mu}+A_{\mu}, \widetilde{A}_{v}\right]-i\left[X_{v}+A_{v}, \widetilde{A}_{\mu}\right]+2 i\left(\left[e_{\mu}^{a}, \omega_{v a}\right]+\left[\omega_{\mu}^{a}, e_{v a}\right]\right)-i \lambda \epsilon_{\mu v}^{\rho} \widetilde{A}_{\rho} .
\end{aligned}
$$

Finally, the action proposed is of Chern-Simons type:

$$
S=\operatorname{Tr} i \epsilon^{\mu v \rho} \mathcal{X}_{\mu} \mathcal{R}_{v \rho}
$$

Variation of the above action leads to the equations of motion:

$$
T_{\mu v}^{a}=0, \quad R_{\mu v}^{a}=0, \quad F_{\mu v}=0 \quad \widetilde{F}_{\mu \nu}=0 .
$$

It is worth noting that all the above results in the construction of the 3-d gravity as a noncommutative gauge theory reduce to the ones obtained in the continuous case (Section 6.2). 


\section{4-d Gravity as a Gauge Theory on Noncommutative Spaces}

In this section, we review the construction of a 4-d gravity model as a noncommutative gauge theory [129]. First, we need to present the construction of a suitable 4-d fuzzy space, on which the gravity model is constructed and then we present the features of the gravity model on this 4- $\mathrm{d}$ fuzzy space.

\subsection{Fuzzy de Sitter Space}

The 4-d fuzzy space that is employed in this task is the fuzzy version of 4-d de Sitter space, $\mathrm{dS}_{4}$, which is defined as a submanifold of the 5-d Minkowski spacetime in a similar way that the four-sphere is considered as an embedding in the 5-d Euclidean space. Specifically, the embedding equation that defines $\mathrm{dS}_{4}$ is:

$$
\eta^{A B} x_{A} x_{B}=R^{2},
$$

where $A, B=0, \ldots, 4$ and $\eta^{A B}$ is the mostly positive metric tensor of the 5-d Minkowski spacetime, i.e., $\eta^{A B}=\operatorname{diag}(-1,+1,+1,+1,+1)$. To obtain the fuzzy analog of this space, one has to consider its coordinates, $X_{a}$, to be operators that do not commute with each other:

$$
\left[X_{a}, X_{b}\right]=i \theta_{a b}
$$

As in the fuzzy sphere case, in which the coordinates were identified as the three rescaled generators of SU(2) in an (large) N-dimensional representation with radius $r$, the right hand side of the above equation, Equation (105), should also be identified as a generator of the algebra, ensuring covariance, i.e., $\theta_{a b}=C_{a b c} X_{c}$, where $C_{a b c}$ is a rescaled Levi-Civita symbol. However, such an identification in this fuzzy de Sitter case is problematic, meaning that identification of the coordinate operators with some generators of $\mathrm{SO}(1,4)$ would break covariance, since the algebra would not be closing, i.e., $\theta_{a b}$ cannot be assigned to generators into the algebra [118] (For a more detailed explanation, see [120,198], in which the same problem is encountered in the construction of the fuzzy four-sphere.). Demanding the preservation of covariance leads to the consideration of a group with larger symmetry, in which all generators identified to the coordinates but also the noncommutativity can be incorporated in it. The minimum enlargement of the symmetry leads to the $\mathrm{SO}(1,5)$ group. Therefore, a fuzzy version of the $\mathrm{dS}_{4}$ space, with coordinates represented by $\mathrm{N}$-dimensional matrices, respecting covariance, too, is obtained after the extension of the symmetry to the $\mathrm{SO}(1,5)$ and, since we use the Euclidean signature to facilitate the construction, the resulting symmetry group is the $\mathrm{SO}(6)$.

To formulate the above 4-d fuzzy space, we consider the generators of the $\mathrm{SO}(6)$ algebra, denoted by $J_{A B}=-J_{B A}$, with $A, B=1, \ldots, 6$, satisfying the following commutation relation:

$$
\left[J_{A B}, J_{C D}\right]=i\left(\delta_{A C} J_{B D}+\delta_{B D} J_{A C}-\delta_{B C} J_{A D}-\delta_{A D} J_{B C}\right) .
$$

Decomposition of the above generators in an $\mathrm{SO}(4)$ notation and identification of the component generators leads to the following definitions:

$$
J_{m n}=\frac{1}{\hbar} \Theta_{m n}, \quad J_{m 5}=\frac{1}{\lambda} X_{m}, \quad J_{m 6}=\frac{\lambda}{2 \hbar} P_{m}, \quad J_{56}=\frac{1}{2} \mathrm{~h},
$$

with $m=1, \ldots, 4$. For dimensional reasons, an elementary length, $\lambda$, has been introduced. The coordinates, momenta and noncommutativity tensors are denoted as $X_{m}, P_{m}$ and $\Theta_{m n}$, respectively. The coordinates and momenta satisfy the following commutation relations:

$$
\begin{gathered}
{\left[X_{m}, X_{n}\right]=i \frac{\lambda^{2}}{\hbar} \Theta_{m n}, \quad\left[P_{m}, P_{n}\right]=4 i \frac{\hbar}{\lambda^{2}} \Theta_{m n}} \\
{\left[X_{m}, P_{n}\right]=i \hbar \delta_{m n} \mathrm{~h}, \quad\left[X_{m}, \mathrm{~h}\right]=i \frac{\lambda^{2}}{\hbar} P_{m}}
\end{gathered}
$$




$$
\left[P_{m}, \mathrm{~h}\right]=4 i \frac{\hbar}{\lambda^{2}} X_{m}
$$

where $m, n=1, \cdots, 4$. The algebra of spacetime transformations is:

$$
\begin{gathered}
{\left[X_{m}, \Theta_{n p}\right]=i \hbar\left(\delta_{m p} X_{n}-\delta_{m n} X_{p}\right)} \\
{\left[P_{m}, \Theta_{n p}\right]=i \hbar\left(\delta_{m p} P_{n}-\delta_{m n} P_{p}\right)} \\
{\left[\Theta_{m n}, \Theta_{p q}\right]=i \hbar\left(\delta_{m p} \Theta_{n q}+\delta_{n q} \Theta_{m p}-\delta_{n p} \Theta_{m q}-\delta_{m q} \Theta_{n p}\right)} \\
{\left[\mathrm{h}, \Theta_{m n}\right]=0 .}
\end{gathered}
$$

The algebra defined above, in contrast to the Heisenberg algebra (see [199]), admits finite dimensional representations for the operators $X_{m}, P_{m}$ and $\Theta_{m n}$, therefore the model of spacetime we obtained is in fact a finite quantum system. In analogy to the fuzzy sphere case, spaces such as the above fuzzy $\mathrm{dS}_{4}$ are called fuzzy covariant spaces $[118,119,200]$.

\subsection{A Noncommutative Gauge Theory of 4-d Gravity}

In this section, we present the formulation of a 4-d gravity model as a gauge theory on the fuzzy $\mathrm{dS}_{4}$ space constructed in the previous section (Section 8.1) (For a string theory approach of a noncommutative gravity model, see [201]). As in the 3-d translation of the gauge-theoretic description of gravity as a gauge theory on the fuzzy space $\mathbb{R}_{\lambda}^{3}$ in Section 7 , the whole procedure is carried out as a noncommutative analog of the cases mentioned in Sections 6.1 and 6.3.

\subsubsection{Determination of the Gauge Group and Representation by $4 \times 4$ Matrices}

Recalling the previous section in which the fuzzy $\mathrm{dS}_{4}$ space was constructed, the symmetry group of the space in which we were led due to preservation of covariance, is the $\mathrm{SO}(6)$. Drawing lessons from the commutative case, in which the isometry group (the Poincaré group) was gauged, in this case the gauge group will be the isometry group of the fuzzy $\mathrm{dS}_{4}$ space, i.e., the $\mathrm{SO}(5)$, viewed as a subgroup of the resulting $\mathrm{SO}(6)$ group.

However, the same problem that emerged in the 3-d case related to the anti-commutators of the generators of the algebra is encountered in this case, too [123,124,196] (see also [95]). The anti-commutators do not yield operators that belong to the algebra and this is exactly the case for the generators of $\mathrm{SO}(5)$. For this reason, the representation of the generators gets fixed and the operators produced by the anti-commutators are included into the algebra, considering them as generators, too. This results in the extension of the initial, $\mathrm{SO}(5)$, gauge group to the $\mathrm{SO}(6) \times \mathrm{U}(1)(\sim \mathrm{U}(4))$ group with the representation of the generators being the 4 of $\mathrm{SO}(6)$ [129].

The starting point is to consider the four Euclidean $\Gamma$-matrices, satisfying the following anti-commutation relation:

$$
\left\{\Gamma_{a}, \Gamma_{b}\right\}=2 \delta_{a b} \mathbf{1}
$$

where $a, b=1, \ldots 4$. In addition, the $\Gamma_{5}$ operator is defined as $\Gamma_{5}=\Gamma_{1} \Gamma_{2} \Gamma_{3} \Gamma_{4}$. Therefore, the generators of the $\mathrm{SO}(6)$ are written as:

(a) six generators of the Lorentz transformations: $\mathrm{M}_{a b}=-\frac{i}{4}\left[\Gamma_{a}, \Gamma_{b}\right]=-\frac{i}{2} \Gamma_{a} \Gamma_{b}, a<b$;

(b) four generators for the conformal boosts: $\mathrm{K}_{a}=\frac{1}{2} \Gamma_{a}$;

(c) four generators for the local translations: $\mathrm{P}_{a}=-\frac{i}{2} \Gamma_{a} \Gamma_{5}$;

(d) one generator for special conformal transformations: $\mathrm{D}=-\frac{1}{2} \Gamma_{5}$; and

(e) one $\mathrm{U}(1)$ generator: $\mathbf{1}$. 
The $\Gamma$-matrices are determined in terms of the Pauli matrices:

$$
\sigma_{1}=\left(\begin{array}{ll}
0 & 1 \\
1 & 0
\end{array}\right) \quad \sigma_{2}=\left(\begin{array}{cc}
0 & -i \\
i & 0
\end{array}\right) \quad \sigma_{3}=\left(\begin{array}{cc}
1 & 0 \\
0 & -1
\end{array}\right)
$$

as tensor products, specifically:

$$
\begin{gathered}
\Gamma_{1}=\sigma_{1} \otimes \sigma_{1}, \quad \Gamma_{2}=\sigma_{1} \otimes \sigma_{2}, \quad \Gamma_{3}=\sigma_{1} \otimes \sigma_{3} \\
\Gamma_{4}=\sigma_{2} \otimes \mathbf{1}, \quad \Gamma_{5}=\sigma_{3} \otimes \mathbf{1} .
\end{gathered}
$$

Therefore, the generators of the algebra are represented by the following $4 \times 4$ matrices:

$$
M_{i j}=-\frac{i}{2} \Gamma_{i} \Gamma_{j}=\frac{1}{2} \mathbf{1} \otimes \sigma_{k}
$$

where $i, j, k=1,2,3$ and:

$$
M_{4 k}=-\frac{i}{2} \Gamma_{4} \Gamma_{k}=-\frac{1}{2} \sigma_{3} \otimes \sigma_{k}
$$

and satisfy the following commutation relations, obtained by straightforward calculations, using the above definitions:

$$
\begin{aligned}
& {\left[K_{a}, K_{b}\right]=i M_{a b}, \quad\left[P_{a}, P_{b}\right]=i M_{a b}} \\
& {\left[X_{a}, P_{b}\right]=i \delta_{a b} D, \quad\left[X_{a}, D\right]=i P_{a}} \\
& {\left[P_{a}, D\right]=i K_{a}, \quad\left[K_{a}, P_{b}\right]=i \delta_{a b} D, \quad\left[K_{a}, D\right]=-i P_{a}} \\
& {\left[K_{a}, M_{b c}\right]=i\left(\delta_{a c} K_{b}-\delta_{a b} K_{c}\right)} \\
& {\left[P_{a}, M_{b c}\right]=i\left(\delta_{a c} P_{b}-\delta_{a b} P_{c}\right)} \\
& {\left[M_{a b}, M_{c d}\right]=i\left(\delta_{a c} M_{b d}+\delta_{b d} M_{a c}-\delta_{b c} M_{a d}-\delta_{a d} M_{b c}\right)} \\
& {\left[D, M_{a b}\right]=0 .}
\end{aligned}
$$

\subsubsection{Noncommutative Gauge Theory of Gravity}

Now that the algebra of the gauge group is determined, it is time to proceed with the noncommutative gauging procedure. First, the covariant coordinate of the theory is defined as:

$$
\hat{X}_{m}=X_{m} \otimes \mathbf{1}+A_{m}(X) .
$$

The coordinate, $\hat{X}_{m}$, defined above is covariant by construction, i.e., the gauge transformation acting on it is covariant:

$$
\delta \hat{X}_{m}=i\left[\epsilon, \hat{X}_{m}\right]
$$

where $\epsilon(X)$ is the gauge parameter, being a function of the coordinates-matrices, $X_{m}$, but, at the same time, takes values in the $\mathrm{SO}(6) \times \mathrm{U}(1)$ algebra. Thus, it may be expressed as a decomposition on the generators of the algebra, explicitly:

$$
\epsilon=\epsilon_{0}(X) \otimes \mathbf{1}+\xi^{a}(X) \otimes K_{a}+\widetilde{\epsilon}_{0}(X) \otimes D+\lambda_{a b}(X) \otimes \Sigma^{a b}+\widetilde{\xi}^{a}(X) \otimes P_{a} .
$$

Taking into consideration that a gauge transformation does not "see" the coordinate $X_{m}$, i.e., $\delta X_{m}=0$, the transformation property of the $A_{m}$ included in Equation (121) is obtained. In accordance with the commutative case, $A_{m}$ transforms in such a way that admits the interpretation of a gauge potential or, in other words, the connection of the gauge theory. Similar to the case of the gauge transformation parameter, $\epsilon$, the connection, $A_{m}$, is a function of the matrices-coordinates $X_{m}$ of the fuzzy space $\mathrm{dS}_{4}$, but also takes values in the $\mathrm{SO}(6) \times \mathrm{U}(1)$ algebra, which means that it can be expanded on its sixteen generators as follows:

$$
A_{m}(X)=e_{m}^{a}(X) \otimes P_{a}+\omega_{m}^{a b}(X) \otimes \Sigma_{a b}(X)+b_{m}^{a}(X) \otimes K_{a}(X)+\widetilde{a}_{m}(X) \otimes D+a_{m}(X) \otimes \mathbf{1},
$$


where it is manifest that one gauge field has been introduced for each generator. The component gauge fields are functions of the coordinates of the space, $X_{m}$, therefore they are $\mathrm{N} \times \mathrm{N}$ matrices, where $\mathrm{N}$ is the dimension of the representation of the coordinates. Thus, instead of the ordinary product, between the gauge fields and the corresponding generators, the tensor product is employed, since the factors are matrices of different dimensions; the generators are $4 \times 4$ matrices. Therefore, every term of the connection is a $4 \mathrm{~N} \times 4 \mathrm{~N}$ matrix.

After the introduction of the gauge fields, the covariant coordinate is now written as:

$$
\hat{X}_{m}=X_{m} \otimes \mathbf{1}+e_{m}^{a}(X) \otimes P_{a}+\omega_{m}^{a b}(X) \otimes \Sigma_{a b}+b_{m}^{a} \otimes K_{a}+\widetilde{a}_{m} \otimes D+a_{m} \otimes \mathbf{1},
$$

The next step is to calculate the field strength tensor for this $\mathrm{SO}(6) \times \mathrm{U}(1)$ noncommutative gauge theory, which, for the fuzzy de Sitter space, is defined as:

$$
\mathcal{R}_{m n}=\left[\hat{X}_{m}, \hat{X}_{n}\right]-\frac{i \lambda^{2}}{\hbar} \hat{\Theta}_{m n}
$$

where $\hat{\Theta}_{m n}=\Theta_{m n} \otimes \mathbf{1}+\mathcal{B}_{m n} . \mathcal{B}_{m n}$ is a non-Abelian two-form gauge field, which takes values in the $\mathrm{SO}(6) \times \mathrm{U}(1)$ algebra. The two-form field was introduced to render the field strength tensor as covariant, since in its absence the field strength tensor does not transform in a covariant way (For details on this generic issue encountered on such spaces, see Appendix A of ref. [129].). These two-form gauge fields that were introduced in the theory contribute with a kinetic term of the following type:

$$
\mathcal{S}_{\mathcal{B}}=\operatorname{Tr} \operatorname{tr} \hat{\mathcal{H}}_{m n p} \hat{\mathcal{H}}^{m n p}
$$

in the complete action that is determined below. Of course, $\hat{\mathcal{H}}_{m n p}$ transforms covariantly under a gauge transformation and therefore the above action is gauge invariant.

The defined field strength tensor, Equation (125), can be expanded in terms of the component curvatures since it is valued in the algebra, i.e.

$$
\mathcal{R}_{m n}(X)=R_{m n}{ }^{a b}(X) \otimes \Sigma_{a b}+\widetilde{R}_{m n}{ }^{a}(X) \otimes P_{a}+R_{m n}{ }^{a}(X) \otimes K_{a}+\widetilde{R}_{m n}(X) \otimes D+R_{m n}(X) \otimes \mathbf{1} .
$$

Now, the necessary information for the determination of the transformations of the gauge fields and the expressions of the component curvature tensors are obtained. For the calculations and results, see Appendix B of [129].

\subsection{The Constraints for the symmetry breaking and the action}

The desired gauge symmetry is the one described by the Lorentz group, in our signature, the $\mathrm{SO}(4)$. A straightforward way to do this is to consider a constrained theory in which the only surviving curvature tensor would be the one associated with the Lorentz generators of the algebra, while the rest would vanish, breaking the initial $\mathrm{SO}(6) \times \mathrm{U}(1)$ symmetry to the $\mathrm{SO}(4) \times \mathrm{U}(1)$. However, following this approach would lead to an over-constrained theory, judging from counting the degrees of freedom. Therefore, it would be wiser to follow a procedure in which the breaking would occur in a less straightforward way. The first constraint in this direction would be:

$$
\widetilde{R}_{m n}^{a}(P)=0,
$$

which is the torsionless condition, and is also considered in the cases where the Einstein and conformal gravity theories are described as (ordinary) gauge theories. In turn, the gauge field $b_{m}^{a}$ admits an interpretation of a second vielbein of the theory, which would lead to a theory with two metrics, which is not desirable in our case. Thus, as a solution of the constraints, the relation $e_{m}^{a}=b_{m}^{a}$ is considered. Taking into consideration the torsionless condition, Equation (128), along with this solution, the correct degrees of freedom are obtained. This also leads to the expression of the spin connection 
$\omega_{m}^{a b}$ in terms of the rest of the fields, $e_{m}^{a}, a_{m}, \widetilde{a}_{m}$. To specify the expression of the spin connection with respect to the rest of the fields, the following two identities have to be employed:

$$
\delta_{f g h}^{a b c}=\epsilon^{a b c d} \epsilon_{f g h d} \quad \text { and } \quad \frac{1}{3 !} \delta_{f g h}^{a b c} a^{f g h}=a^{[f g h]} .
$$

The constraint of zero torsion, $\widetilde{R}(P)=0$, takes the following form:

$$
\epsilon^{a b c d}\left[e_{m}^{b}, \omega_{n}^{c d}\right]-i\left\{\omega_{m}^{a b}, e_{n b}\right\}=-\left[D_{m}, e_{m}^{a}\right]-i\left\{e_{m}^{a}, \widetilde{a}_{m}\right\},
$$

where $D_{m}=X_{m}+a_{m}$, i.e., the covariant coordinate of an Abelian gauge theory. The above equation leads to the following two:

$$
\epsilon^{a b c d}\left[e_{m}^{b}, \omega_{n}^{c d}\right]=-\left[D_{m}, e_{m}^{a}\right] \text { and }\left\{\omega_{m}^{a b}, e_{n b}\right\}=\left\{e_{m}^{a}, \widetilde{a}_{n}\right\} .
$$

Making use of above the identities, Equation (129), the above equations lead to the expression of the spin connection in terms of the rest of the fields:

$$
\omega_{n}^{a c}=-\frac{3}{4} e_{b}^{m}\left(-\epsilon^{a b c d}\left[D_{m}, e_{n d}\right]+\delta^{[b c}\left\{e_{n}^{a]}, \widetilde{a}_{m}\right\}\right) .
$$

Now, according to ref. [202], the vanishing of the field strength tensors in a gauge theory would lead to the vanishing of the corresponding gauge field. However, this cannot be applied in this case, since $\widetilde{R}(P)=0$ would mean $e_{\mu}^{a}=0$, which would lead to degeneracy of the metric tensor of the space [91]. The field that can be set to zero is the $\widetilde{a}_{m}$. This fixing will also modify the expression of the spin connection, Equation (132), leading to an even simpler expression:

$$
\omega_{n}{ }^{a c}=\frac{3}{4} e^{m}{ }_{b} \epsilon^{a b c d}\left[D_{m}, e_{n d}\right] .
$$

At this point, it should be noted that the $\mathrm{U}(1)$ field strength tensor, $R_{m n}(\mathbf{1})$, is not considered to be vanishing, which means that this $\mathrm{U}(1)$, which is strongly related to the noncommutativity, remains unbroken in the resulting theory after the symmetry breaking, since the theory still describes gravity on a noncommutative space. However, its corresponding field, $a_{m}$, would vanish if the commutative limit of the broken theory, in which noncommutativity is lifted and $a_{m}$ decouples, were considered. In this limit, the gauge symmetry would be just $\mathrm{SO}(4)$.

Another way of resulting with the desired $\mathrm{SO}(4)$ gauge symmetry as a remnant symmetry after the breaking of $\mathrm{SO}(6)$ is to extrapolate to the noncommutative case the argument developed for the conformal gravity in the commutative case, including two scalars in the 6 representation of $\mathrm{SO}(6)$. It is expected that the spontaneous symmetry breaking induced by the scalars would lead to some constraints, such as the ones in Equation (128).

In addition, the commutative limit of the above $\mathrm{SO}(6) \times \mathrm{U}(1)$ gauge theory leads to a theory with $\mathrm{SO}(6)$ gauge symmetry, since the gauge field, $a_{m}$, is related to the $\mathrm{U}(1)$ part of the symmetry decouples. Therefore, all expressions obtained in the noncommutative gauge theory (gauge connection, transformations of the fields, and component curvature tensors) reduce to the corresponding ones of the gauge-theoretic description of the conformal group (in the Euclidean signature) of Section 6.3.

\subsection{The Action and Equations of Motion}

The most reasonable choice for the action is that of Yang-Mills form (A Yang-Mills action $\operatorname{tr} F^{2}$ on the fuzzy de Sitter space is gauge invariant; for details, see Appendix A of [129]):

$$
\mathcal{S}=\operatorname{Trtr}\left\{\mathcal{R}_{m n}, \mathcal{R}_{r s}\right\} \epsilon^{m n r s},
$$


where $\operatorname{Tr}$ is the trace over the matrices-coordinates (takes the role of the integration of the continuous case) while tr is the trace over the algebra. Considering the vacuum state of the theory, after the symmetry breaking, the surviving terms of the action would be:

$$
\mathcal{S}=2 \operatorname{Tr}\left(R_{m n}^{a b} R_{r s}^{c d} \epsilon_{a b c d} \epsilon^{m n r s}+4 \widetilde{R}_{m n} R_{r s} \epsilon^{m n r s}+\frac{1}{3} H_{m n p}^{a b} H^{m n p c d} \epsilon_{a b c d}+\frac{4}{3} \widetilde{H}_{m n p} H^{m n p}\right),
$$

where the above expression of the action with indices in the $\operatorname{SO}(4)$ notation $(a, b, c, d=1, \ldots 4)$ was obtained by starting with the action expressed in the $\mathrm{SO}(5)$ notation $(A, B, C, D=1, \ldots, 5)$ for the gauge indices, calculating the trace of the generators which filtered out most of the terms and. then decomposing the surviving terms to the desired $\mathrm{SO}(4)$ notation.

Replacing with the explicit expressions of the component tensors and expressing the $\omega$ gauge field in terms of the rest of the surviving gauge fields, Equation (133), then variation with respect to the (surviving) gauge fields would lead to the equations of motion.

\section{Conclusions}

Starting from Kaluza-Klein theory, the foundations were set for the construction of 4-d gauge theories from geometric origins. In this direction, phenomenologically viable particle physics models have been constructed, starting from gauge theories in higher dimensions and making use of the CSDR scheme (with support from the heterotic string). Moreover, to start with renormalizable higher-dimensional theories and obtain 4-d renormalizable theories too, fuzzy coset spaces were employed to represent the extra dimensions. In addition, to provide support in these scenarios, one could start with a 4-d $\mathcal{N}=4$ supersymmetric Yang-Mills theory with a suitable particle content and generate the results of a dimensional reduction of a higher-dimensional initial theory with fuzzy extra dimensions. This picture would lead to an $\mathcal{N}=1$ GUT and subsequently to extensions of the MSSM after a symmetry breaking.

Furthermore, aiming at a more complete picture of the description of the interactions, gravity models were also accommodated in the framework of noncommutativity, making use of the noncommutative gauge theories. In this concept, it was reasonable to translate the already established description of the gauge-theoretic description of 3-d and 4-d gravity to the noncommutative regime, giving insight into its behavior in high-energy scales (Planck scale) in which coordinates are naturally considered to be noncommutative. For this purpose, certain covariant fuzzy spaces with suitable isometries were employed and the models that were built on them included interesting results and found contact with low-energy gravity theories.

From our perspective, our work of the four-dimensional noncommutative gravity model contributes greatly, mainly in two aspects. The first is that we have accomplished a successful construction of a four-dimensional covariant fuzzy space, in such a way that can be generalized for other spaces, specifically by enlarging the symmetry and introducing a two-form gauge field. Second, we managed to give a description of the gravitational interaction in a regime in which the coordinates can be considered as noncommutative (e.g., Planck scale), and relate it with the conformal gravity in the commutative, low-energy limit. Our priority in the future is to study the Lorentz invariant action we obtained and attempt to relate it with the four-dimensional Einstein-Hilbert action, with aspirations of connecting the large and low-energy regimes for the gravitational interaction. Then, after obtaining the equations of motion, we plan to explore potential applications in cosmology.

Author Contributions: The three authors of the present article contributed equally in each part of the research.

Funding: This research was partially funded by the COST Action MP1405 for two of us (GM and GZ). One of us (GZ) has been supported within the Excellence Initiative funded by the German and States Governments, at the Institute for Theoretical Physics, Heidelberg University and from the Excellent Grant Enigmass of LAPTh.

Acknowledgments: We would like to thank Ali Chamseddine, Paolo Aschieri, Thanassis Chatzistavrakidis, Evgeny Ivanov, Larisa Jonke, Danijel Jurman, Alexandros Kehagias, Dieter Lüst, Denjoe O'Connor, Emmanuel Saridakis, Harold Steinacker, Kelly Stelle, Patrizia Vitale and Christof Wetterich for useful discussions. Two of us 
(GM and GZ) would like to thank ESI-Vienna for the hospitality during their participation in the Workshop "Matrix Models for Noncommutative Geometry and String Theory", 9-13 July 2018. G.Z. would like to thank the ITP-Heidelberg, LAPTh—Annecy and MPI—Munich for their hospitality.

Conflicts of Interest: The authors declare no conflict of interest. The funders had no role in the design of the study; in the collection, analyses, or interpretation of data; in the writing of the manuscript, or in the decision to publish the results.

\section{References}

1. Green, M.B.; Schwarz, J.H.; Witten, E. Superstring Theory. Vol. 1: Introduction; Cambridge Monographs on Mathematical Physics; Cambridge University Press: Cambridge, UK, 1987; 469p.

2. Gross, D.J.; Harvey, J.A.; Martinec, E.J.; Rohm, R. Heterotic String Theory. 1. The Free Heterotic String. Nucl. Phys. B 1985, 256, 253-284. [CrossRef]

3. Forgacs, P.; Manton, N.S. Space-Time Symmetries in Gauge Theories. Commun. Math. Phys. 1980, 72, 15-35. [CrossRef]

4. Kapetanakis, D.; Zoupanos, G. Coset space dimensional reduction of gauge theories. Phys. Rep. 1992, 219, 4-76. [CrossRef]

5. Kubyshin, Y.A.; Volobuev, I.P.; Mourao, J.M.; Rudolph, G. Dimensional Reduction of Gauge Theories, Spontaneous Compactification and Model Building. Lect. Notes Phys. 1990, 349, 1. [CrossRef]

6. Scherk, J.; Schwarz, J.H. How to Get Masses from Extra Dimensions. Nucl. Phys. B 1979, 153, 61-88. [CrossRef]

7. Manton, N.S. Fermions and Parity Violation in Dimensional Reduction Schemes. Nucl. Phys. B 1981, 193, 502-516. [CrossRef]

8. Chapline, G.; Slansky, R. Dimensional Reduction and Flavor Chirality. Nucl. Phys. B 1982, 209, 461-483. [CrossRef]

9. Candelas, P.; Horowitz, G.T.; Strominger, A.; Witten, E. Vacuum Configurations for Superstrings. Nucl. Phys. B 1985, 258, 46-74. [CrossRef]

10. Cardoso, G.L.; Curio, G.; Dall'Agata, G.; Lust, D.; Manousselis, P.; Zoupanos, G. NonKahler string backgrounds and their five torsion classes. Nucl. Phys. B 2003, 652, 5-34. [CrossRef]

11. Strominger, A. Superstrings with Torsion. Nucl. Phys. B 1986, 274, 253-284. [CrossRef]

12. Lust, D. Compactification of Ten-dimensional Superstring Theories Over Ricci Flat Coset Spaces. Nucl. Phys. B 1986, 276, 220-240. [CrossRef]

13. Castellani, L.; Lust, D. Superstring Compactification on Homogeneous Coset Spaces with Torsion. Nucl. Phys. B 1988, 296, 143-156. [CrossRef]

14. Becker, K.; Becker, M.; Dasgupta, K.; Green, P.S. Compactifications of heterotic theory on nonKahler complex manifolds, 1. JHEP 2003, 0304, 007. [CrossRef]

15. Becker, K.; Becker, M.; Green, P.S.; Dasgupta, K.; Sharpe, E. Compactifications of heterotic strings on nonKahler complex manifolds, 2. Nucl. Phys. B 2004, 678, 19-100. [CrossRef]

16. Gurrieri, S.; Lukas, A.; Micu, A. Heterotic on half-flat. Phys. Rev. D 2004, 70, 126009. [CrossRef]

17. Benmachiche, I.; Louis, J.; Martinez-Pedrera, D. The Effective action of the heterotic string compactified on manifolds with SU(3) structure. Class. Quantum Gravity 2008, 25, 135006. [CrossRef]

18. Micu, A. Heterotic compactifications and nearly-Kahler manifolds. Phys. Rev. D 2004, 70, 126002. [CrossRef]

19. Frey, A.R.; Lippert, M. AdS strings with torsion: Non-complex heterotic compactifications. Phys. Rev. D 2005, 72, 126001. [CrossRef]

20. Manousselis, P.; Prezas, N.; Zoupanos, G. Supersymmetric compactifications of heterotic strings with fluxes and condensates. Nucl. Phys. B 2006, 739, 85-105. [CrossRef]

21. Chatzistavrakidis, A.; Manousselis, P.; Zoupanos, G. Reducing the Heterotic Supergravity on nearly-Kahler coset spaces. Fortschr. Phys. 2009, 57, 527-534. [CrossRef]

22. Chatzistavrakidis, A.; Zoupanos, G. Dimensional Reduction of the Heterotic String over nearly-Kaehler manifolds. J. High Energy Phys. 2009, 0909, 077. [CrossRef]

23. Dolan, B.P.; Szabo, R.J. Dimensional Reduction and Vacuum Structure of Quiver Gauge Theory. J. High Energy Phys. 2009, 0908, 038. [CrossRef]

24. Lechtenfeld, O.; Nolle, C.; Popov, A.D. Heterotic compactifications on nearly Kahler manifolds. J. High Energy Phys. 2010, 1009, 074. [CrossRef] 
25. Popov, A.D.; Szabo, R.J. Double quiver gauge theory and nearly Kahler flux compactifications. J. High Energy Phys. 2012, 1202, 033. [CrossRef]

26. Klaput, M.; Lukas, A.; Matti, C. Bundles over Nearly-Kahler Homogeneous Spaces in Heterotic String Theory. J. High Energy Phys. 2011, 1109, 100. [CrossRef]

27. Chatzistavrakidis, A.; Lechtenfeld, O.; Popov, A.D. Nearly Káhler heterotic compactifications with fermion condensates. J. High Energy Phys. 2012, 1204, 114. [CrossRef]

28. Gray, J.; Larfors, M.; Lust, D. Heterotic domain wall solutions and SU(3) structure manifolds. J. High Energy Phys. 2012, 1208, 099. [CrossRef]

29. Klaput, M.; Lukas, A.; Matti, C.; Svanes, E.E. Moduli Stabilising in Heterotic Nearly Káhler Compactifications. J. High Energy Phys. 2013, 1301, 015. [CrossRef]

30. Irges, N.; Zoupanos, G. Reduction of $N=1, E_{8} S Y M$ over $S U(3) / U(1) \times U(1) \times Z_{3}$ and its four-dimensional effective action. Phys. Lett. B 2011, 698, 146-151. [CrossRef]

31. Irges, N.; Orfanidis, G.; Zoupanos, G. Dimensional Reduction of N=1, $E_{8}$ SYM over SU(3)/U(1) x U(1) x Z and its four-dimensional effective action. POS CORFU 2011, 2011, 105. [CrossRef]

32. Butruille, J.-B. Homogeneous nearly Kähler manifolds. arXiv 2006, arXiv:math.DG/0612655.

33. Manousselis, P.; Zoupanos, G. Soft supersymmetry breaking due to dimensional reduction over nonsymmetric coset spaces. Phys. Lett. B 2001, 518, 171-180. [CrossRef]

34. Manousselis, P.; Zoupanos, G. Supersymmetry breaking by dimensional reduction over coset spaces. Phys. Lett. B 2001, 504, 122-130. [CrossRef]

35. Manousselis, P.; Zoupanos, G. Dimensional reduction of ten-dimensional supersymmetric gauge theories in the $\mathrm{N}=1, \mathrm{D}=4$ superfield formalism. J. High Energy Phys. 2004, 0411, 025. [CrossRef]

36. Connes, A. Noncommutative Geometry; Academic Press, Inc.: San Diego, CA, USA, 1994.

37. Madore, J. An Introduction to Noncommutative Differential Geometry and Its Physical Applications; London Mathematical Society Lecture Note Series; Cambridge University Press: Cambridge, UK, 1999; Vol. 257.

38. Madore, J. The Fuzzy sphere. Class. Quantum Gravity 1992, 9, 69-88. [CrossRef]

39. Buric, M.; Grammatikopoulos, T.; Madore, J.; Zoupanos, G. Gravity and the structure of noncommutative algebras. J. High Energy Phys. 2006, 0604, 054. [CrossRef]

40. Filk, T. Divergencies in a field theory on quantum space. Phys. Lett. B 1996, 376, 53-58. [CrossRef]

41. Varilly, J.C.; Gracia-Bondia, J.M. On the ultraviolet behavior of quantum fields over noncommutative manifolds. Int. J. Mod. Phys. A 1999, 14, 1305. [CrossRef]

42. Chaichian, M.; Demichev, A.; Presnajder, P. Quantum field theory on noncommutative space-times and the persistence of ultraviolet divergences. Nucl. Phys. B 2000, 567, 360-390. [CrossRef]

43. Minwalla, S.; Raamsdonk, M.V.; Seiberg, N. Noncommutative perturbative dynamics. J. High Energy Phys. 2000, 0002, 020. [CrossRef]

44. Grosse, H.; Wulkenhaar, R. Renormalization of phi**4 theory on noncommutative $\mathrm{R}^{* *} 4$ to all orders. Lett. Math. Phys. 2005, 71, 13-26. [CrossRef]

45. Grosse, H.; Steinacker, H. Exact renormalization of a noncommutative phi ${ }^{* *} 3$ model in 6 dimensions. Adv. Theor. Math. Phys. 2008, 12, 605-639. [CrossRef]

46. Grosse, H.; Steinacker, H. Finite gauge theory on fuzzy CP**2. Nucl. Phys. B 2005, 707, 145-198. [CrossRef]

47. Connes, A.; Lott, J. Particle Models and Noncommutative Geometry (Expanded Version). Nucl. Phys. Proc. Suppl. 1991, 18B, 29-47. [CrossRef]

48. Chamseddine, A.H.; Connes, A. The Spectral Action Principle. Commun. Math. Phys. 1997, $186,731$. [CrossRef]

49. Chamseddine, A.H.; Connes, A. Conceptual Explanation for the Algebra in the Noncommutative Approach to the Standard Model. Phys. Rev. Lett. 2007, 99, 191601. [CrossRef] [PubMed]

50. Martin, C.P.; Gracia-Bondia, J.M.; Varilly, J.C. The Standard model as a noncommutative geometry: The Low-energy regime. Phys. Rep. 1998, 294, 363-406. [CrossRef]

51. Dubois-Violette, M.; Madore, J.; Kerner, R. Gauge Bosons in a Noncommutative Geometry. Phys. Lett. B 1989, 217, 485-488. [CrossRef]

52. Dubois-Violette, M.; Madore, J.; Kerner, R. Classical Bosons in a Noncommutative Geometry. Class. Quantum Gravity 1989, 6, 1709. [CrossRef]

53. Dubois-Violette, M.; Kerner, R.; Madore, J. Noncommutative Differential Geometry and New Models of Gauge Theory. J. Math. Phys. 1990, 31, 323. [CrossRef] 
54. Madore, J. On a quark-lepton duality. Phys. Lett. B 1993, 305, 84-89. [CrossRef]

55. Madore, J. On a noncommutative extension of electrodynamics. Fundam. Theor. Phys. 1993, 52, $285-298$.

56. Connes, A.; Douglas, M.R.; Schwarz, A.S. Noncommutative geometry and matrix theory: Compactification on tori. J. High Energy Phys. 1998, 9802, 003. [CrossRef]

57. Seiberg, N.; Witten, E. String theory and noncommutative geometry. J. High Energy Phys. 1999, 9909, 032. [CrossRef]

58. Ishibashi, N.; Kawai, H.; Kitazawa, Y.; Tsuchiya, A. A Large N reduced model as superstring. Nucl. Phys. B 1997, 498, 467-491. [CrossRef]

59. Jurco, B.; Schraml, S.; Schupp, P.; Wess, J. Enveloping algebra valued gauge transformations for nonAbelian gauge groups on noncommutative spaces. Eur. Phys. J. C 2000, 17, 521-526. [CrossRef]

60. Jurco, B.; Schupp, P.; Wess, J. NonAbelian noncommutative gauge theory via noncommutative extra dimensions. Nucl. Phys. B 2001, 604, 148-180. [CrossRef]

61. Jurco, B.; Moller, L.; Schraml, S.; Schupp, P.; Wess, J. Construction of nonAbelian gauge theories on noncommutative spaces. Eur. Phys. J. C 2001, 21, 383-388. [CrossRef]

62. Barnich, G.; Brandt, F.; Grigoriev, M. Seiberg-Witten maps and noncommutative Yang-Mills theories for arbitrary gauge groups. J. High Energy Phys. 2002, 0208, 023. [CrossRef]

63. Chaichian, M.; Presnajder, P.; Sheikh-Jabbari, M.M.; Tureanu, A. Noncommutative standard model: Model building. Eur. Phys. J. C 2003, 29, 413-432. [CrossRef]

64. Calmet, X.; Jurco, B.; Schupp, P.; Wess, J.; Wohlgenannt, M. The Standard model on noncommutative space-time. Eur. Phys. J. C 2002, 23, 363-376. [CrossRef]

65. Aschieri, P.; Jurco, B.; Schupp, P.; Wess, J. Noncommutative GUTs, standard model and C,P,T. Nucl. Phys. B 2003, 651, 45-70. [CrossRef]

66. Behr, W.; Deshpande, N.G.; Duplancic, G.; Schupp, P.; Trampetic, J.; Wess, J. The Z $\longrightarrow$ gamma gamma, g g decays in the noncommutative standard model. Eur. Phys. J. C 2003, 29, 441-447. [CrossRef]

67. Aschieri, P.; Madore, J.; Manousselis, P.; Zoupanos, G. Dimensional reduction over fuzzy coset spaces. J. High Energy Phys. 2004, 0404, 034. [CrossRef]

68. Aschieri, P.; Madore, J.; Manousselis, P.; Zoupanos, G. Unified theories from fuzzy extra dimensions. Fortschr. Phys. 2004, 52, 718-723. [CrossRef]

69. Aschieri, P.; Madore, J.; Manousselis, P.; Zoupanos, G. Renormalizable theories from fuzzy higher dimensions. arXiv 2004, arXiv:hep-th/0503039.

70. Aschieri, P.; Grammatikopoulos, T.; Steinacker, H.; Zoupanos, G. Dynamical generation of fuzzy extra dimensions, dimensional reduction and symmetry breaking. J. High Energy Phys. 2006, 0609, 026. [CrossRef]

71. Aschieri, P.; Steinacker, H.; Madore, J.; Manousselis, P.; Zoupanos, G. Fuzzy Extra Dimensions: Dimensional Reduction, Dynamical Generation and Renormalizability. SFIN A 2007, arXiv:0704.28801, 25.

72. Steinacker, H.; Zoupanos, G. Fermions on spontaneously generated spherical extra dimensions. J. High Energy Phys. 2007, 0709, 017. [CrossRef]

73. Chatzistavrakidis, A.; Steinacker, H.; Zoupanos, G. On the fermion spectrum of spontaneously generated fuzzy extra dimensions with fluxes. Fortschr. Phys. 2010, 58, 537-552. [CrossRef]

74. Chatzistavrakidis, A.; Steinacker, H.; Zoupanos, G. Orbifolds, fuzzy spheres and chiral fermions. J. High Energy Phys. 2010, 1005, 100. [CrossRef]

75. Chatzistavrakidis, A.; Zoupanos, G. Higher-Dimensional Unified Theories with Fuzzy Extra Dimensions. SIGMA 2010, 6, 063. [CrossRef]

76. Gavriil, D.; Manolakos, G.; Orfanidis, G.; Zoupanos, G. Higher-Dimensional Unification with continuous and fuzzy coset spaces as extra dimensions. Fortschr. Phys. 2015, 63, 442-467. [CrossRef]

77. Manolakos, G.; Zoupanos, G. The trinification model SU(3) ${ }^{3}$ from orbifolds for fuzzy spheres. Phys. Part. Nucl. Lett. 2017, 14, 322-327. [CrossRef]

78. Manolakos, G.; Zoupanos, G. Higher-Dimensional Unified Theories with continuous and fuzzy coset spaces as extra dimensions. Springer Proc. Math. Stat. 2016, 191, 203-229. [CrossRef]

79. Utiyama, R. Invariant theoretical interpretation of interaction. Phys. Rev. 1956, 101, 1597. [CrossRef]

80. Kibble, T.W.B. Lorentz invariance and the gravitational field. J. Math. Phys. 1961, 2, 212. [CrossRef]

81. Stelle, K.S.; West, P.C. Spontaneously Broken De Sitter Symmetry and the Gravitational Holonomy Group. Phys. Rev. D 1980, 21, 1466. [CrossRef] 
82. MacDowell, S.W.; Mansouri, F. Unified Geometric Theory of Gravity and Supergravity. Phys. Rev. Lett. 1977, 38, 739; Erratum in 1977, 38, 1376. [CrossRef]

83. Ivanov, E.A.; Niederle, J. Gauge Formulation of Gravitation Theories. 1. The Poincare, De Sitter and Conformal Cases. In Proceedings of the 9th International Colloquium on Group Theoretical Methods in Physics, Cocoyoc, Mexico, 23-27 June 1980; C80-06-23.3, pp. 545-551. Phys. Rev. D 1982, 25, 976. [CrossRef]

84. Ivanov, E.A.; Niederle, J. Gauge Formulation of Gravitation Theories. 2. The Special Conformal Case. Phys. Rev. D 1982, 25, 988. [CrossRef]

85. Kibble, T.W.B.; Stelle, K.S. Gauge theories of gravity and supergravity. In Progress In Quantum Field Theory; Ezawa, H., Kamefuchi, S., Eds.; pp. 57-81.

86. Kaku, M.; Townsend, P.K.; van Nieuwenhuizen, P. Gauge Theory of the Conformal and Superconformal Group. Phys. Lett. 1977, 69B, 304-308. [CrossRef]

87. Fradkin, E.S.; Tseytlin, A.A. Conformal Supergravity. Phys. Rep. 1985, 119, 233. [CrossRef]

88. Freedman, D.Z.; Proeyen, A.V. Supergravity; Cambridge University Press: Cambridge, UK, 2012.

89. Chamseddine, A.H. Supersymmetry and Higher Spin Fields. Ph.D. Thesis, Department of Theoretical Physics Imperial, College of Science and Technology, London, UK, 1976.

90. Chamseddine, A.H.; West, P.C. Supergravity as a Gauge Theory of Supersymmetry. Nucl. Phys. B 1977, 129, 39-44. [CrossRef]

91. Witten, E. (2+1)-Dimensional Gravity as an Exactly Soluble System. Nucl. Phys. B 1988, 311, 46-78. [CrossRef]

92. Madore, J.; Schraml, S.; Schupp, P.; Wess, J. Gauge theory on noncommutative spaces. Eur. Phys. J. C 2000, 16, 161-167. [CrossRef]

93. Chamseddine, A.H. Deforming Einstein's gravity. Phys. Lett. B 2001, 504, 33-37. [CrossRef]

94. Chamseddine, A.H. SL(2,C) gravity with complex vierbein and its noncommutative extension. Phys. Rev. D 2004, 69, 024015. [CrossRef]

95. Aschieri, P.; Castellani, L. Noncommutative D=4 gravity coupled to fermions. J. High Energy Phys. 2009, 0906, 086. [CrossRef]

96. Aschieri, P.; Castellani, L. Noncommutative supergravity in $\mathrm{D}=3$ and $\mathrm{D}=4$. J. High Energy Phys. 2009, 0906, 087. [CrossRef]

97. Ćirić, M.D.; Nikolić, B.; Radovanović, V. Noncommutative $S O(2,3) \star$ gravity: Noncommutativity as a source of curvature and torsion. Phys. Rev. D 2017, 96, 064029. [CrossRef]

98. Cacciatori, S.; Klemm, D.; Martucci, L.; Zanon, D. Noncommutative Einstein-AdS gravity in three-dimensions. Phys. Lett. B 2002, 536, 101. [CrossRef]

99. Cacciatori, S.; Chamseddine, A.H.; Klemm, D.; Martucci, L.; Sabra, W.A.; Zanon, D. Noncommutative gravity in two dimensions. Class. Quantum Gravity 2002, 19, 4029. [CrossRef]

100. Aschieri, P.; Castellani, L. Noncommutative Chern-Simons gauge and gravity theories and their geometric Seiberg-Witten map. J. High Energy Phys. 2014, 1411, 103. [CrossRef]

101. Banados, M.; Chandia, O.; Grandi, N.E.; Schaposnik, F.A.; Silva, G.A. Three-dimensional noncommutative gravity. Phys. Rev. D 2001, 64, 084012. [CrossRef]

102. Banks, T.; Fischler, W.; Shenker, S.H.; Susskind, L. M theory as a matrix model: A Conjecture. Phys. Rev. D 1997, 55, 5112. [CrossRef]

103. Aoki, H.; Iso, S.; Kawai, H.; Kitazawa, Y.; Tada, T. Space-time structures from IIB matrix model. Prog. Theor. Phys. 1998, 99, 713-746. [CrossRef]

104. Hanada, M.; Kawai, H.; Kimura, Y. Describing curved spaces by matrices. Prog. Theor. Phys. 2006, 114, 1295-1316. [CrossRef]

105. Furuta, K.; Hanada, M.; Kawai, H.; Kimura, Y. Field equations of massless fields in the new interpretation of the matrix model. Nucl. Phys. B 2007, 767, 82-99. [CrossRef]

106. Yang, H.S. Emergent Gravity from Noncommutative Spacetime. Int. J. Mod. Phys. A 2009, 24, 4473-4517. [CrossRef]

107. Steinacker, H. Emergent Geometry and Gravity from Matrix Models: An Introduction. Class. Quantum Gravity 2010, 27, 133001. [CrossRef]

108. Kim, S.W.; Nishimura, J.; Tsuchiya, A. Expanding (3+1)-dimensional universe from a Lorentzian matrix model for superstring theory in (9+1)-dimensions. Phys. Rev. Lett. 2012, 108, 011601. [CrossRef] [PubMed]

109. Nishimura, J. The Origin of space-time as seen from matrix model simulations. PTEP 2012, $2012,01 \mathrm{~A} 101$. [CrossRef] 
110. Nair, V.P. Gravitational fields on a noncommutative space. Nucl. Phys. B 2003, 651, 313-327. [CrossRef]

111. Abe, Y.; Nair, V.P. Noncommutative gravity: Fuzzy sphere and others. Phys. Rev. D 2003, 68, 025002. [CrossRef]

112. Valtancoli, P. Gravity on a fuzzy sphere. Int. J. Mod. Phys. A 2004, 19, 361-370. [CrossRef]

113. Nair, V.P. The Chern-Simons one-form and gravity on a fuzzy space. Nucl. Phys. B 2006, 750, $321-333$. [CrossRef]

114. Burić, M.; Madore, J.; Zoupanos, G. WKB Approximation in Noncommutative Gravity. SIGMA 2007, 3, 125. [CrossRef]

115. Burić, M.; Madore, J.; Zoupanos, G. The Energy-momentum of a Poisson structure. Eur. Phys. J. C 2008, 55, 489-498. [CrossRef]

116. Snyder, H.S. Quantized space-time. Phys. Rev. 1947, 71, 38. [CrossRef]

117. Yang, C.N. On quantized space-time. Phys. Rev. 1947, 72, 874. [CrossRef]

118. Heckman, J.; Verlinde, H. Covariant non-commutative space-time. Nucl. Phys. B 2015, 894, 58-74. [CrossRef]

119. Burić, M.; Madore, J. Noncommutative de Sitter and FRW spaces. Eur. Phys. J. C 2015, 75, 502. [CrossRef]

120. Sperling, M.; Steinacker, H.C. Covariant 4-dimensional fuzzy spheres, matrix models and higher spin. J. Phys. A 2017, 50, 375202. [CrossRef]

121. Burić, M.; Latas, D.; Nenadovixcx, L. Fuzzy de Sitter Space. arXiv 2017, arXiv:1709.05158. [CrossRef]

122. Steinacker, H.C. Emergent gravity on covariant quantum spaces in the IKKT model. J. High Energy Phys. 2016, 1612, 156. [CrossRef]

123. Chatzistavrakidis, A.; Jonke, L.; Jurman, D.; Manolakos, G.; Manousselis, P.; Zoupanos, G. Noncommutative Gauge Theory and Gravity in Three Dimensions. Fortschr. Phys. 2018, 66, 1800047. [CrossRef]

124. Jurman, D.; Manolakos, G.; Manousselis, P.; Zoupanos, G. Gravity as a Gauge Theory on Three-Dimensional Noncommutative spaces. PoS CORFU 2018, 2017, 162. [CrossRef]

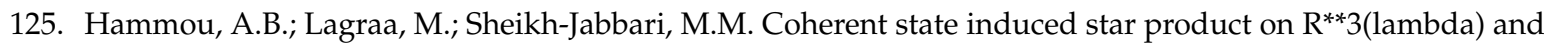
the fuzzy sphere. Phys. Rev. D 2002, 66, 025025. [CrossRef]

126. Vitale, P. Noncommutative field theory on $\mathbb{R}_{\lambda}^{3}$. Fortschr. Phys. 2014, 62, 825. [CrossRef]

127. Kováčik, S.; Prešnajder, P. The velocity operator in quantum mechanics in noncommutative space. J. Math. Phys. 2013, 54, 102103. [CrossRef]

128. Jurman, D.; Steinacker, H. 2D fuzzy Anti-de Sitter space from matrix models. J. High Energy Phys. 2014, 1401, 100. [CrossRef]

129. Manolakos, G.; Manousselis, P.; Zoupanos, G. Four-dimensional Gravity on a Covariant Noncommutative Space. arXiv 2019, arXiv:1902.10922.

130. Kaluza, T. Zum Unitätsproblem der Physik. Sitzungsber. Preuss. Akad. Wiss. Berl. (Math. Phys.) 1921, 1921, 966-972, Translation, Int. J. Mod. Phys. D 2018, 27, 1870001. [CrossRef]

131. Klein, O. Quantum Theory and Five-Dimensional Theory of Relativity. (In German and English). Z. Phys. 1926, 37, 895-906, Surv. High Energ. Phys. 1986, 5, 241-244. [CrossRef]

132. Kerner, R. Generalization of the Kaluza-Klein Theory for an Arbitrary Nonabelian Gauge Group. Ann. Inst. Henr. Poincare Phys. Theor. 1968, 9, 143-152.

133. Cho, Y.M. Higher - Dimensional Unifications of Gravitation and Gauge Theories. J. Math. Phys. 2006, 16, 2029-2035. [CrossRef]

134. Cho, Y.M.; Freund, P.G.O. Nonabelian Gauge Fields in Nambu-Goldstone Fields. Phys. Rev. D 1975, $12,1711$. [CrossRef]

135. Mecklenburg, W. The Kaluza-Klein Idea: Status and Prospects. Fortschr. Phys. 1984, 32, 207-260. [CrossRef]

136. Bailin, D.; Love, A. Kaluza-klein Theories. Rep. Prog. Phys. 1987, 50, 1087. [CrossRef]

137. Salam, A.; Strathdee, J.A. On Kaluza-Klein Theory. Ann. Phys. 1982, 141, 316-352. [CrossRef]

138. Witten, E. Fermion Quantum Numbers Kaluza-Klein Theory. Conf. Proc. C 1983, 8306011, 227.

139. Horvath, Z.; Palla, L.; Cremmer, E.; Scherk, J. Grand Unified Schemes and Spontaneous Compactification. Nucl. Phys. B 1977, 127, 57-65. [CrossRef]

140. Wetterich, C. Dimensional Reduction of Weyl, Majorana and Majorana-weyl Spinors. Nucl. Phys. B 1983, 222, 20-44. [CrossRef]

141. Palla, L. On Dimensional Reduction of Gauge Theories: Symmetric Fields and Harmonic Expansion. Z. Phys. C 1984, 24, 195. [CrossRef] 
142. Pilch, K.; Schellekens, A.N. Formulae for the Eigenvalues of the Laplacian on Tensor Harmonics on Symmetric Coset Spaces. J. Math. Phys. 1984, 25, 3455. [CrossRef]

143. Forgacs, P.; Horvath, Z.; Palla, L. Spontaneous Compactification To Nonsymmetric Spaces. Z. Phys. C 1986, 30, 261-266. [CrossRef]

144. Chapline, G.; Manton, N.S. The Geometrical Significance of Certain Higgs Potentials: An Approach to Grand Unification. Nucl. Phys. B 1981, 184, 391-405. [CrossRef]

145. Bais, F.A.; Barnes, K.J.; Forgacs, P.; Zoupanos, G. Dimensional Reduction of Gauge Theories Yielding Unified Models Spontaneously Broken to SU(3) X U(1). Nucl. Phys. B 1986, 263, 557-590. [CrossRef]

146. Kubyshin, Y.A.; Mourao, J.M.; Volobuev, I.P. Scalar Fields in the Dimensional Reduction Scheme for Symmetric Spaces. Int. J. Mod. Phys. A 1989, 4, 151-171. [CrossRef]

147. Harnad, J.P.; Shnider, S.; Vinet, L. The Yang-Mills System in Compactified Minkowski Space. 1. Invariance Conditions and SU(2) Invariant Solutions. J. Math. Phys. 1979, 20, 931. [CrossRef]

148. Harnad, J.P.; Vinet, L.; Shnider, S. Group Actions on Principal Bundles and Invariance Conditions for Gauge Fields. J. Math. Phys. 1980, 21, 2719. [CrossRef]

149. Harnad, J.P.; Shnider, S.; Tafel, J. Group Actions on Principal Bundles and Dimensional Reduction. Lett. Math. Phys. 1980, 4, 107-113. [CrossRef]

150. Farakos, K.; Koutsoumbas, G.; Surridge, M.; Zoupanos, G. Dimensional Reduction and the Higgs Potential. Nucl. Phys. B 1987, 291, 128-140. [CrossRef]

151. Farakos, K.; Koutsoumbas, G.; Surridge, M.; Zoupanos, G. Geometrical Hierarchy and Spontaneous Symmetry Breaking. Phys. Lett. B 1987, 191, 135-140. [CrossRef]

152. Barnes, K.J.; Forgacs, P.; Surridge, M.; Zoupanos, G. On Fermion Masses in a Dimensional Reduction Scheme. Z. Phys. C 1987, 33, 427-431. [CrossRef]

153. Harland, D.; Kurkcuoglu, S. Equivariant reduction of Yang-Mills theory over the fuzzy sphere and the emergent vortices. Nucl. Phys. B 2009, 821, 380-398. [CrossRef]

154. Arkani-Hamed, N.; Cohen, A.G.; Georgi, H. Electroweak symmetry breaking from dimensional deconstruction. Phys. Lett. B 2001, 513, 232. [CrossRef]

155. Arkani-Hamed, N.; Cohen, A.G.; Georgi, H. (De)constructing dimensions. Phys. Rev. Lett. 2001, 86, 4757. [CrossRef]

156. Andrews, R.P.; Dorey, N. Deconstruction of the Maldacena-Nunez compactification. Nucl. Phys. B 2006, 751, 304-341. [CrossRef]

157. Kachru, S.; Silverstein, E. 4-D conformal theories and strings on orbifolds. Phys. Rev. Lett. 1998, 80, 4855. [CrossRef]

158. Steinacker, H. Quantized gauge theory on the fuzzy sphere as random matrix model. Nucl. Phys. B 2004, 679, 66-98. [CrossRef]

159. Kim, J.E. Z(3) orbifold construction of $S U(3)^{* * 3}$ GUT with $\sin ^{* * 2}($ theta0(W)) $=3 / 8$. Phys. Lett. B 2003, 564, 35-41. [CrossRef]

160. Choi, K.S.; Kim, J.E. Three family Z(3) orbifold trinification, MSSM and doublet triplet splitting problem. Phys. Lett. B 2003, 567, 87-92. [CrossRef]

161. Maalampi, J.; Roos, M. Physics of Mirror Fermions. Phys. Rep. 1990, 186, 53. [CrossRef]

162. Bailin, D.; Love, A. Orbifold compactifications of string theory. Phys. Rep. 1999, 315, 285-408. [CrossRef]

163. Douglas, M.R.; Greene, B.R.; Morrison, D.R. Orbifold resolution by D-branes. Nucl. Phys. B 1997, 506, 84-106. [CrossRef]

164. Aldazabal, G.; Ibanez, L.E.; Quevedo, F.; Uranga, A.M. D-branes at singularities: A Bottom up approach to the string embedding of the standard model. J. High Energy Phys. 2000, 0008, 002. [CrossRef]

165. Lawrence, A.E.; Nekrasov, N.; Vafa, C. On conformal field theories in four-dimensions. Nucl. Phys. B 1998, 533, 199-209. [CrossRef]

166. Kiritsis, E. D-branes in standard model building, gravity and cosmology. Phys. Rep. 2005, 421, 105-190, Erratum in 2006, 429, 121-122. [CrossRef]

167. Sohnius, M.F. Introducing Supersymmetry. Phys. Rep. 1985, 128, 39-204. [CrossRef]

168. Brink, L.; Schwarz, J.H.; Scherk, J. Supersymmetric Yang-Mills Theories. Nucl. Phys. B 1977, 121, 77-92. [CrossRef]

169. Gliozzi, F.; Scherk, J.; Olive, D.I. Supersymmetry, Supergravity Theories and the Dual Spinor Model. Nucl. Phys. B 1977, 122, 253-290. [CrossRef] 
170. Djouadi, A. The Anatomy of electro-weak symmetry breaking. II. The Higgs bosons in the minimal supersymmetric model. Phys. Rep. 2008, 459, 1-241. [CrossRef]

171. Steinacker, H. Gauge theory on the fuzzy sphere and random matrices. Springer Proc. Phys. 2005, 98, 307-311. [CrossRef]

172. Grosse, H.; Lizzi, F.; Steinacker, H. Noncommutative gauge theory and symmetry breaking in matrix models. Phys. Rev. D 2010, 81, 085034. [CrossRef]

173. Steinacker, H. Emergent Gravity and Noncommutative Branes from Yang-Mills Matrix Models. Nucl. Phys. B 2009, 810, 1-39. [CrossRef]

174. Glashow, S.L. Trinification of All Elementary Particle Forces. In Proceedings of the Fifth Workshop on Grand Unification, Providence, Rhode Island, 12-14 April 1984; Print-84-0577 (BOSTON).

175. Rizov, V.A. A Gauge Model of the Electroweak and Strong Interactions Based on the Group SU(3) $)_{L} X \mathrm{SU}(3)_{R}$ X SU(3) . Bulg. J. Phys. 1981, 8, 461-477.

176. Heinemeyer, S.; Ma, E.; Mondragon, M.; Zoupanos, G. Finite SU(3)**3 model. AIP Conf. Proc. 2010, 1200, 568. [CrossRef]

177. Ma, E.; Mondragon, M.; Zoupanos, G. Finite SU(N)**k unification. J. High Energy Phys. 2004, $0412,026$. [CrossRef]

178. Lazarides, G.; Panagiotakopoulos, C. MSSM from SUSY trinification. Phys. Lett. B 1994, 336, $190-193$. [CrossRef]

179. Babu, K.S.; He, X.G.; Pakvasa, S. Neutrino Masses and Proton Decay Modes in SU(3) X SU(3) X SU(3) Trinification. Phys. Rev. D 1986, 33, 763. [CrossRef]

180. Leontaris, G.K.; Rizos, J. A D-brane inspired $\mathrm{U}(3)(\mathrm{C}) \times \mathrm{U}(3)(\mathrm{L}) \times \mathrm{U}(3)(\mathrm{R})$ model. Phys. Lett. B 2006, 632, 710-716. [CrossRef]

181. Heinemeyer, S.; Mondragon, M.; Zoupanos, G. The LHC Higgs boson discovery: Implications for Finite Unified Theories. Int. J. Mod. Phys. A 2014, 29, 1430032. [CrossRef]

182. Heinemeyer, S.; Mondragon, M.; Tracas, N.; Zoupanos, G. Reduction of Couplings in Quantum Field Theories with applications in Finite Theories and the MSSM. Springer Proc. Math. Stat. 2014, 111, 177. [CrossRef]

183. Heinemeyer, S.; Mondragon, M.; Zoupanos, G. Finite Unification: Theory and Predictions. SIGMA 2010, 6, 049. [CrossRef]

184. Mondragon, M.; Zoupanos, G. Finite unified theories: Theoretical basis and phenomenological implications. Phys. Part. Nucl. Lett. 2011, 8, 173. [CrossRef]

185. Heinemeyer, S.; Mondragón, M.; Tracas, N.; Zoupanos, G. Reduction of Couplings and its application in Particle Physics. arXiv 2019, arXiv:1904.00410. [CrossRef]

186. Chatzistavrakidis, A.; Steinacker, H.; Zoupanos, G. Orbifold matrix models and fuzzy extra dimensions. In Proceedings of the School and Workshops on Elementary Particle Physics and Gravity, Corfu, Greece, 4-18 September 2011. [CrossRef]

187. Chatzistavrakidis, A.; Steinacker, H.; Zoupanos, G. Intersecting branes and a standard model realization in matrix models. J. High Energy Phys. 2011, 1109, 115. [CrossRef]

188. Chepelev, I.; Makeenko, Y.; Zarembo, K. Properties of D-branes in matrix model of IIB superstring. Phys. Lett. B 1997, 400, 43. [CrossRef]

189. Fayyazuddin, A.; Smith, D.J. P-brane solutions in IKKT IIB matrix theory. Mod. Phys. Lett. A 1997, 12, 1447-1454. [CrossRef]

190. Aoki, H.; Ishibashi, N.; Iso, S.; Kawai, H.; Kitazawa, Y.; Tada, T. Noncommutative Yang-Mills in IIB matrix model. Nucl. Phys. B 2000, 565, 176-192. [CrossRef]

191. Iso, S.; Kimura, Y.; Tanaka, K.; Wakatsuki, K. Noncommutative gauge theory on fuzzy sphere from matrix model. Nucl. Phys. B 2001, 604, 121-147. [CrossRef]

192. Kimura, Y. Noncommutative gauge theories on fuzzy sphere and fuzzy torus from matrix model. Prog. Theor. Phys. 2001, 106, 445. [CrossRef]

193. Kitazawa, Y. Matrix models in homogeneous spaces. Nucl. Phys. B 2002, 642, 210-226. [CrossRef]

194. Aoki, H.; Iso, S.; Suyama, T. Orbifold matrix model. Nucl. Phys. B 2002, 634, 71-89. [CrossRef]

195. Li, L.F. Group Theory of the Spontaneously Broken Gauge Symmetries. Phys. Rev. D 1974, 9, 1723. [CrossRef]

196. Manolakos, G.; Zoupanos, G. Non-commutativity in Unified Theories and Gravity. Springer Proc. Math. Stat. 2017, 263, 177-198. [CrossRef] 
197. DeBellis, J.; Saemann, C.; Szabo, R.J. Quantized Nambu-Poisson Manifolds in a 3-Lie Algebra Reduced Model. J. High Energy Phys. 2011, 1104, 075. [CrossRef]

198. Kimura, Y. Noncommutative gauge theory on fuzzy four sphere and matrix model. Nucl. Phys. B 2002, 637, 177-198. [CrossRef]

199. Singh, A.; Carroll, S.M. Modeling Position and Momentum in Finite-Dimensional Hilbert Spaces via Generalized Clifford Algebra. arXiv 2018, arXiv:1806.10134.

200. Barut, A. From Heisenberg algebra to Conformal Dynamical Group. In Conformal Groups and related Symmetries. Physical Results and Mathematical Backgrounf; Lecture Notes in Physics; Barut, A., Doener, H.D., Eds.; Springer: Berlin/Heidelberg, Germany, 1985.

201. Alvarez-Gaume, L.; Meyer, F.; Vazquez-Mozo, M.A. Comments on noncommutative gravity. Nucl. Phys. B 2006, 753, 92-127. [CrossRef]

202. Green, M.B.; Schwarz, J.H.; Witten, E. Superstring Theory. Vol. 2: Loop Amplitudes, Anomalies and Phenomenology; Cambridge Monographs On Mathematical Physics; Cambridge University Press: Cambridge, UK, 1987; 596p.

(C) 2019 by the authors. Licensee MDPI, Basel, Switzerland. This article is an open access article distributed under the terms and conditions of the Creative Commons Attribution (CC BY) license (http://creativecommons.org/licenses/by/4.0/). 